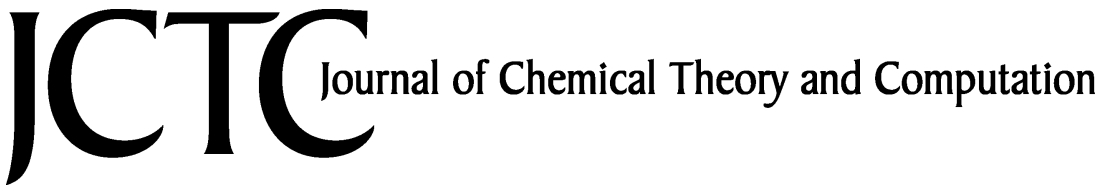

\section{Theoretical Investigation of Excited States of Oligothiophenes and of Their Monocations}

\author{
Ulrike Salzner* \\ Bilkent University, Department of Chemistry, 06800 Bilkent, Ankara, Turkey \\ Received November 27, 2006
}

\begin{abstract}
Excitation energies of neutral thiophene oligomers with chain lengths of up to 25 rings and charged thiophene oligomers with chain lengths of up to 20 rings were calculated with time-dependent Hartree-Fock and time-dependent density functional theory (TDDFT). As recently for polyene cations, very good agreement is found between TDDFT and high-level ab initio calculations and with experimental results wherever data are available. For short thiophene oligomer cations, two sub-band transitions are predicted; for long chains, a third transition develops. Defects are found to be delocalized in bare cations; the inclusion of counterions induces localization. Despite the strong influence of counterions on the geometry, the influence of counterions on the spectra is small for the first two sub-band peaks. Since counterions are directly involved in the electron transitions contributing to the third sub-band peak of longer oligomers, the inclusion of counterions lowers the energy of this absorption peak. The agreement between theoretical spectra based on delocalized geometries and experimental spectra shows that defect localization (electron phonon coupling) is not the underlying cause of the two subband transitions. Investigation of the electronic configurations that contribute to the excited states does not confirm the nature of the transitions predicted with the polaron model.
\end{abstract}

\section{Introduction}

Polythiophene (PT) is an organic semiconductor that increases its conductivity by several orders of magnitude upon oxidation (p-doping). ${ }^{1}$ During the doping process, the strong $\pi-\pi^{*}$ absorption of the neutral polymer in the UV spectrum decreases in intensity and shifts to higher energy. At the same time, two new features start developing at lower energy. ${ }^{2}$ Doping is therefore associated with a shift in the absorption energy and causes a color change. The evolution of the absorption peaks is crucial for understanding electrochromic properties and for characterizing the charge carriers produced during doping.

The doping process of organic polymers differs from that of inorganic semiconductors since oxidation of organic molecules causes geometry and band structure changes. A widely accepted model for rationalizing the doping process is that at low doping levels polarons form, which combine into bipolarons as the doping proceeds. ${ }^{3-7}$ Polarons are

\footnotetext{
* E-mail: salzner@fen.bilkent.edu.tr.
}

radical cations that consist of the combined distortion of electronic and geometric structures (electron-phonon coupling). The associated distortion from aromatic to quinoid structure is generally believed to be "self-localized" over a short chain segment because for neutral PT the quinoid form is higher in energy than the aromatic form. Therefore, the cation is believed to keep the quinoid chain segment as short as possible. Bipolarons are dications that consist of two positive charges that are separated by a quinoid chain segment. For bipolarons, self-localization results from a competition between electronic repulsion between the two like charges and the tendency to minimize the lengths of the quinoid segment. Polarons are associated with three sub-band transitions in the UV/vis spectrum, bipolarons with two. Since doped PT exhibits two features in the UV spectrum and since bipolarons are spinless, the low electron spin resonance (ESR) signal for doped PT was originally considered evidence for bipolaron formation. ${ }^{6,7}$

As solid organic polymers are highly disordered, UV bands are relatively broad and peak assignments are difficult. To 
get around the problems with polydispersity and disorder, many researchers prefer working with well-characterized thiophene oligomers instead of with polymers. ${ }^{8}$ Comparing oligomer with polymer properties revealed that properties of neutral PT are well-reproduced with systems as short as sexi- and octithiophene, ${ }^{9,10}$ although excitation energies of neutral oligothiophenes (OTs) in solution decrease up to a chain length of about 20 rings. Between chain lengths of 20 and 27 rings, excitation energies hardly change, but the intensity of the maximum absorption peak continues to rise. ${ }^{11-14}$ These findings indicate that effective conjugation lengths in PT might be rather short. Therefore, OTs are probably more realistic models for PT than infinitely long polymers.

Similarly, doping experiments of short OTs in solution ${ }^{15-25}$ showed that OT radical cations reproduce spectral properties of PT upon doping. In solution, radical cations could be obtained for bithiophene and longer oligomers, while dications require chain lengths of at least six thiophene rings. In contrast to predictions of the polaron-bipolaron model, radical cations (polarons) exhibit two peaks in the UV spectrum and dications (bipolarons) show only one. ${ }^{16,19-22,24,26-32}$ Thus, doped PT might contain polarons rather than bipolarons. Low ESR signals in doped PT are not inconsistent with polaron formation since interacting polarons also may have singlet ground states. ${ }^{33}$ Especially at low temperatures, cations have a tendency to dimerize. Since dimerization is more likely in a solid than in dilute solution, spinless $\pi$ dimers of radical cations offer an explanation for conductivity in the absence of spins. ${ }^{19-21,24}$ An alternative rationalization for the small ESR signals in doped PT was suggested when experiments revealed that duodecithiophene and longer OTs give rise to UV spectra that are consistent with spinless interacting polaron pairs located on the same chain. ${ }^{32,34,35}$

Theoretical investigations of excited states for bi- and terthiophene cations have been carried out at the complete active space self-consistent field CASSCF ${ }^{36}$ and CASPT2 $2^{37}$ levels of theory. The calculations agree with the experimental solution data and predict two excitations for radical cations. The excitations are multiconfigurational, indicating that the polaron-bipolaron model, which is based on single electron transitions, is oversimplified. Unfortunately, CASPT2 calculations cannot be extended to larger systems. In fact, the active space for the terthiophene cation had to be reduced, since a complete active space containing $17 \pi$ electrons is computationally too demanding. Excited states of longer OTs have been studied with intermediate neglect of differential overlap combined with singles excitations configuration interaction (INDO/SCI) ${ }^{38}$ with the equation of motion coupled cluster (INDO/EOM-CCSD) method, ${ }^{39}$ and with time-dependent density functional theory (TDDFT). ${ }^{40}$ Two transitions were confirmed for polarons in OT monocations.

Evidence is therefore mounting that the polaron-bipolaron model does not fully explain optical properties of conducting polymers upon doping. Therefore, high-level calculations on longer oligomers are needed. In a recent paper, ${ }^{41}$ excited states of polyene cations were investigated with TDDFT. Comparison of TDDFT excitation energies for oligomers up to $\mathrm{C}_{10} \mathrm{H}_{12}{ }^{+}$with multireference perturbation theory (MRMP) values and with experimental results indicated that TDDFT produces reliable excitation energies for short- and mediumsized polyene cations. ${ }^{41}$ Geometry optimizations of longer odd-numbered closed-shell polyene cations with MøllerPlesset perturbation theory truncated at second order (MP2) and with a DFT hybrid functional (B3P86 with 30\% Hartree-Fock exchange) gave very similar results with respect to defect sizes. Comparison of excitation energies of odd-numbered closed-shell polyene cations with timedependent Hartree-Fock (TDHF) theory indicated that TDDFT does not deteriorate for cations in the long chain lengths limit. ${ }^{41}$ Since these results are very encouraging, TDHF and TDDFT are employed here to investigate spectral properties of neutral oligothiophenes and oligothiophene cations.

\section{Methods}

Thiophene oligomers with 2-25 thiophene units were optimized in neutral states and with 2-20 rings in charged states with density functional theory employing the B3P86 hybrid functional ${ }^{42,43}$ with $30 \%$ HF exchange ${ }^{44}$ (B3P86-30\%) and Stevens-Basch-Krauss pseudopotentials with polarized split-valence basis sets (CEP-31G*). ${ }^{45,46}$ The amount of HF exchange in the B3P86 functional was originally increased from $20 \%$ to $30 \%$ to reproduce absorption maxima of polyenes with orbital energy differences. ${ }^{44,47}$ It was shown later that the B3P86-30\%/CEP-31G* level of theory also gives reasonable estimates of band gaps of $\mathrm{PT}$ and polypyrrole $^{44,48}$ and provides accurate structures of polyenes. ${ }^{41}$ Moreover, TDDFT calculations with the B3P86-30\% functional give excellent agreement with experimental results for excited states of polyene cations. ${ }^{41}$ These findings are in agreement with results of other groups that showed that about $20-40 \%$ HF exchange is necessary to produce good geometries, ${ }^{49}$ band gaps,${ }^{50}$ energy level spacings as compared to peak positions in photoelectron spectra, ${ }^{51}$ and vibronic structures of electronic absorption spectra, ${ }^{52,53}$ while the type of exchange functional itself makes little difference. ${ }^{47,53}$

Neutral and charged species were kept planar and obey $C_{2 h}$ (even number of rings) or $C_{2 v}$ (odd number of rings) symmetries. For $6 \mathrm{~T}$, the effect of planarization was evaluated by comparing excitation energies of planar and twisted forms. Radicals were shown to have planar ground states by Zade and Bendikov. ${ }^{40}$ The effect of counterions was probed on $3 \mathrm{~T}-\mathrm{Cl}_{3}, 9 \mathrm{~T}-\mathrm{Cl}_{3}, 13 \mathrm{~T}-\mathrm{Cl}_{3}$, and $19 \mathrm{~T}-\mathrm{Cl}_{3}$ complexes. $\mathrm{Cl}_{3}{ }^{-}$is used as a model for iodine doping. The counterions were placed at the center of the chain and in the plane of the backbone rather than above the chain. In agreement with electrostatics, placement of the anion next to the slightly positively charged hydrogen atoms corresponds to the minimum energy structure. No covalent bond formation as with $\mathrm{Cl}^{-54}$ was observed during structure optimization with $\mathrm{Cl}_{3}{ }^{-}$as the counterion. In contrast to polyene radical cations ${ }^{41}$ there is very little spin contamination for OT radical cations when the B3P86$30 \%$ functional is used. The expectation value for the spin operator for $20 \mathrm{~T}^{+}$is 0.76 . The highest value, 0.84, was obtained for the complexes with $\mathrm{Cl}_{3}{ }^{-}$. There was therefore no need to employ the restricted open-shell formalism. In contrast, HF wave functions are strongly spin-contaminated 
with an expectation value of the spin operator of 1.16 already for the bithiophene cation. Thus, HF and MP2 were not considered for radical cations.

Excited states for neutral species were calculated with TDHF and TDB3P86-30\% employing the CEP-31G* basis set. Studies on polyenes have shown that this basis set is sufficient for calculations of extended systems since no change was observed beyond hexatriene when diffuse functions were added. ${ }^{55}$ Although TDDFT has been shown in numerous studies to provide good excitation spectra, ${ }^{52,56-63}$ there is one major exception. The $1{ }^{1} \mathrm{Bu}$ excited state of large conjugated systems, the very transition that gives rise to the band gap in conducting polymers, is predicted to lie too low in energy. ${ }^{41,53,55,57,59,61,63-70}$ The underestimation of the excitation energy is associated with the fact that the $1{ }^{1} \mathrm{Bu}$ excited state has ionic character in a valence-bond interpretation (compare ref 71 treating polyenes). In general, the underestimation of TDDFT excitation energies for conjugated $\pi$ systems increases with increasing ionic character of the excited state and with increasing size of the system. ${ }^{66}$ For this reason, TDDFT is not employed here for neutral thiophene oligomers. Careful comparison of TDHF excitation energies with high-level ab initio predictions and with experimental spectra of polyenes and of OTs has shown that TDHF reproduces the $1{ }^{1} \mathrm{Bu}$ excitation energies of large conjugated systems very well. ${ }^{41,72-74}$ Thus, TDHF/CEP-31G* is employed here to determine the excitation energies and to define the sub-band region of the neutral species.

In general, TDDFT was shown to be able to tread radicals successfully. ${ }^{58}$ In a recent investigation, it turned out that, despite the failure of TDDFT for the neutral conjugated systems, excited states of polyene cations and radical cations ${ }^{41}$ are reproduced accurately compared to experimental results and compared to MRMP calculations. Grozema et al. observed the same good performance of TDDFT for thienylene vinylene oligomer dications. ${ }^{75}$ Moreover, the quality of the polyene radical cation excited states was found to be independent of the amount of HF exchange since results with the B3P86-30\% and BP86 functionals were almost identical for the first two excited states of polyene cations. Differences start showing, however, for higher-lying excited states. ${ }^{41}$ Since the failure of TDDFT for neutral systems depends on the ionic character of the excited states ${ }^{66}$ it is not surprising that TDDFT performs better for the excited states of radical cations which are quite different in nature ${ }^{71}$ compared to those of the neutral species. OT radical cations were therefore treated with time-dependent pure DFT (TDBP86) and with TDB3P86-30\%. Excitation energies for cations were computed up to the TDHF transition energy of the neutral species. For $20 \mathrm{~T}^{+}$, excitation energies up to the third strong peak were obtained, since for $16 \mathrm{~T}^{+}$and $19 \mathrm{~T}^{+}$ no further sub-band peaks were found. For long oligomer cations, up to 40 excited states had to be considered.

Finally, solvent effects in the presence of dichloromethane were determined for neutral $6 \mathrm{~T}, 2 \mathrm{~T}^{+}, 3 \mathrm{~T}^{+}, 8 \mathrm{~T}^{+}, 9 \mathrm{~T}-\mathrm{Cl}_{3}$, and $13 \mathrm{~T}-\mathrm{Cl}_{3}$ with the polarized continuum model (PCM) as implemented in G03. ${ }^{76-94}$ All calculations are done with Gaussian 03, revision D02. ${ }^{95}$

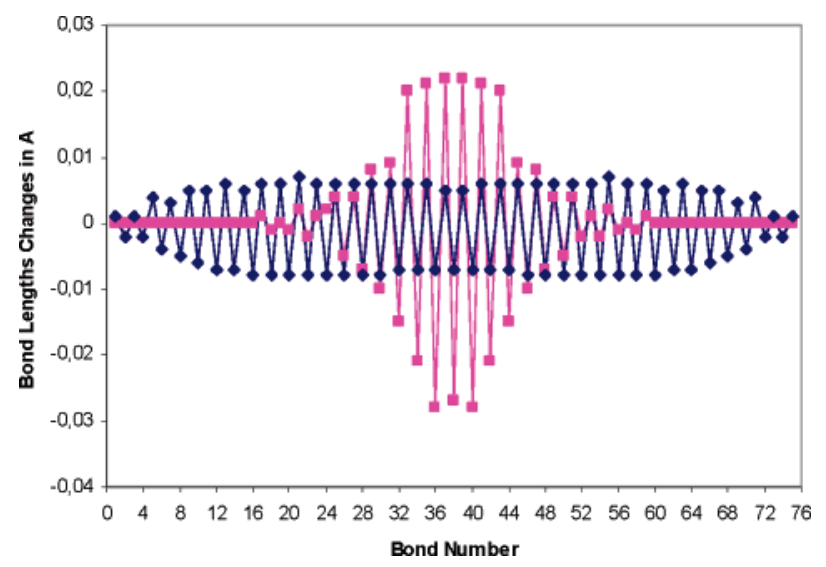

Figure 1. Bond length changes in $19 \mathrm{~T}^{+}$(blue diamonds) and $19 \mathrm{~T}-\mathrm{Cl}_{3}$ (pink squares) compared to neutral 19T. Bond lengths shown are those of $\mathrm{C}-\mathrm{C}$ and $\mathrm{C}=\mathrm{C}$ bonds numbered from the beginning to the end of the chain.

\section{Results}

Geometry of Cations. In agreement with recent studies, ${ }^{75,96-102}$ the $\mathrm{B} 3 \mathrm{P} 86-30 \% / \mathrm{CEP}-31 \mathrm{G}^{*}$ level of theory produces delocalized defects for radical cations in the absence of counterions. In Figure 1, bond length changes in the radical cation in the absence and in the presence of a counterion with respect to the neutral species are plotted for the longest OT investigated here, $19 \mathrm{~T}^{+}$and the $19 \mathrm{~T}-\mathrm{Cl}_{3}$ complex. The geometry distortion in the bare cation is distributed evenly in the middle of the chain. Toward the end, the geometry distortion decreases, with the terminal rings being hardly affected, but no convergence of the defect size with increasing chain length seems to occur. The defect delocalization is not a consequence of spin contamination, since the expectation value for the spin operator for $19 \mathrm{~T}^{+}$is only 0.77 , corresponding closely to a pure doublet with an $\left\langle S^{2}\right\rangle$ value of 0.75 . In the presence of a counterion, the defect is localized over 11 thiophene rings. The main distortion affects the inner three rings, which are quinoid, and falls off quickly over the next four rings. The last four terminal rings are undistorted compared to the neutral system.

The geometries of the bare cation and of the complex with one counterion differ considerably. The energetic effect of these structural differences was evaluated by removing the counterion and performing a single-point calculation on the cation at the geometry of the complex $\left(19 \mathrm{~T}^{+} / / 19 \mathrm{~T}-\mathrm{Cl}_{3}\right)$. The energy difference between the optimized cation and the cation at the geometry of the complex is $1.76 \mathrm{kcal} / \mathrm{mol}(0.09$ $\mathrm{kcal} / \mathrm{mol}$ per ring). Thus, potential energy surfaces of OT radical cations are very flat. This agrees with findings for polyene cations. ${ }^{41}$

The distribution of the positive charge in $19 \mathrm{~T}^{+}, 19 \mathrm{~T}-\mathrm{Cl}_{3}$, and $19 \mathrm{~T}^{+} / / 19 \mathrm{~T}-\mathrm{Cl}_{3}$ is plotted versus the ring number in Figure 2 . In the bare cation, there is a relatively even distribution of the charge over the entire molecule with two slight maxima at rings 5 and 15 and slightly less charge in the middle. For $19 \mathrm{~T}^{+} / / 19 \mathrm{~T}-\mathrm{Cl}_{3}$, a slight shift of the charge toward the middle is observed. This charge shift can be attributed to electron-phonon coupling. In the presence of the counterion, a sharp peak of the positive charge is obtained at the central ring that falls off exponentially toward the chain 


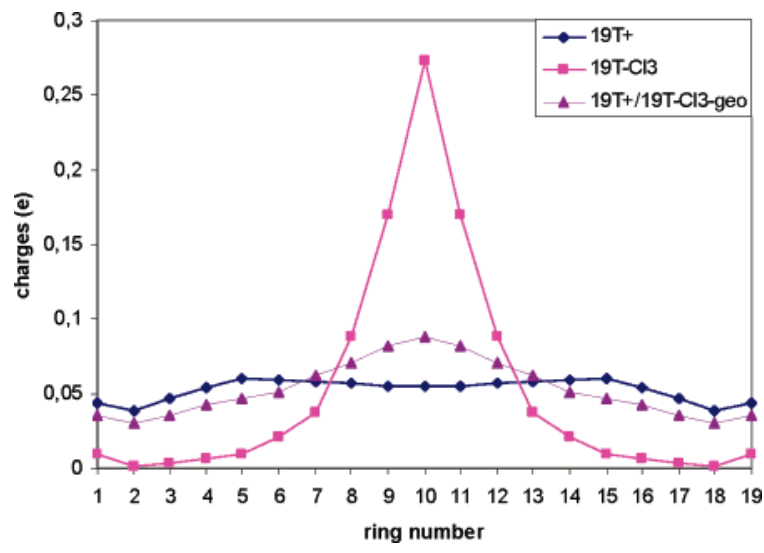

Figure 2. Distribution of the positive charge in $19 \mathrm{~T}^{+}$, in $19-$ $\mathrm{T}^{+}$with the backbone structure of $19 \mathrm{~T}-\mathrm{Cl}_{3}$, and in $19 \mathrm{~T}-\mathrm{Cl}_{3}$. Rings are numbered from the beginning to the end of the chain.

ends. The three species therefore represent polymers with a localized defect $\left(19 \mathrm{~T}-\mathrm{Cl}_{3}\right)$, with a delocalized defect $\left(19 \mathrm{~T}^{+}\right)$, and with a localized structural defect but delocalized charge distribution $\left(19 \mathrm{~T}^{+} / / 19 \mathrm{~T}-\mathrm{Cl}_{3}\right)$.

Excited States. Neutral Thiophene Oligomers. No gasphase data exist for thiophene oligomers. Solvent effects on excitation energies are therefore unknown. Thiophene oligomers absorb and emit at different energies in roomtemperature (RT) solutions; in a matrix, absorption and emissions occur at the same value and coincide with the room-temperature emission. ${ }^{103-106}$ Differences between RT solution and matrix data were attributed to planarization of the twisted thiophene chains upon cooling. To assess influences of the solvent and nonplanarity theoretically, sexithiophene (6T) was optimized at the MP2/CEP-31G* level with planar and nonplanar structures. MP2 was employed since the B3P86-30\%/CEP-31G* level of theory does not produce a nonplanar geometry. The nonplanar form of $6 \mathrm{~T}$ with an optimized twist angle of $25^{\circ}$ is $1.44 \mathrm{kcal} / \mathrm{mol}$ more stable than the planar form. The TDHF excitation energy to the $1{ }^{1} \mathrm{~B}_{\mathrm{u}}$ state of the nonplanar form is $3.04 \mathrm{eV}$, $0.32 \mathrm{eV}$ higher than that of the planar conformation. For the nonplanar form, the excitation energy was also computed in the presence of $\mathrm{CH}_{2} \mathrm{Cl}_{2}$ with the PCM. ${ }^{76-91}$ Inclusion of the solvent lowers the excitation energy by $0.09 \mathrm{eV}$.

By comparison with polyene data, for which experimental solvent effects are $0.3-0.4 \mathrm{eV}^{107}$ and calculated solvent effects are $\sim 0.28 \mathrm{eV},{ }^{74}$ it becomes clear that solvent effects are smaller for thiophene oligomers than for polyenes and that calculated solvent effects are underestimated compared to experimental results. A reasonable estimate is therefore that solvent effects decrease excitation energies by about 0.2 $\mathrm{eV}$. Additional differences with experimental results arise since vertical excitation energies are used, which means that zero-point energy (ZPE) differences between ground and excited states are neglected and that Franck-Condon (FC) factors were not calculated explicitly. Inclusion of the latter two corrections can lead to a lowering of the excitation energy of about $0.3 \mathrm{eV} .{ }^{108}$

Taking the corrections together, $0.2 \mathrm{eV}$ (solvent) +0.3 $\mathrm{eV}(\mathrm{ZPE}$ and FC) $-0.32 \mathrm{eV}$ (planarization) $=0.18 \mathrm{eV}$,

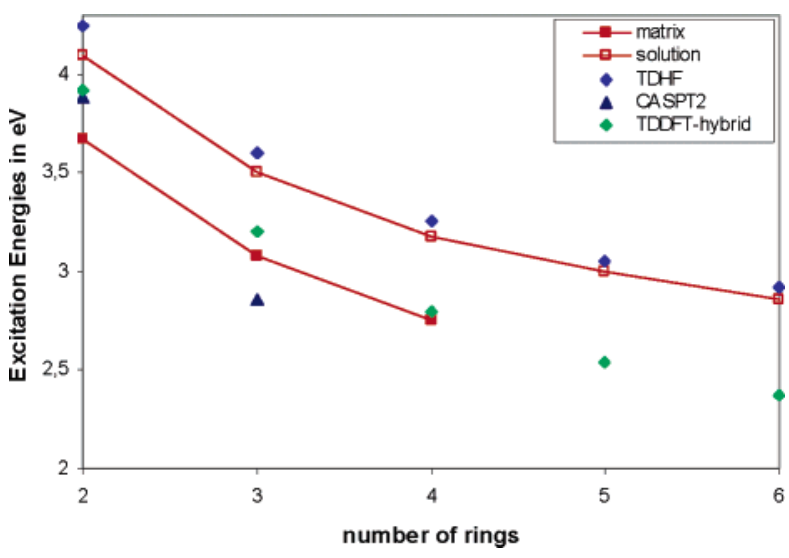

Figure 3. ${ }^{1} \mathrm{~B}_{\mathrm{u}}$ excitation energies ( $\lambda_{\max }$ values) for neutral thiophene oligomers in RT solution, ${ }^{103}$ in matrix, ${ }^{104-106}$ at the CASPT2 level, ${ }^{37}$ at the TDHF/CEP-31G*//B3P86-30\%/CEP$31 \mathrm{G}^{*}$ level, and at the TDB3P86-31G*// B3P86-30\%/CEP$31 \mathrm{G}^{*}$ level.

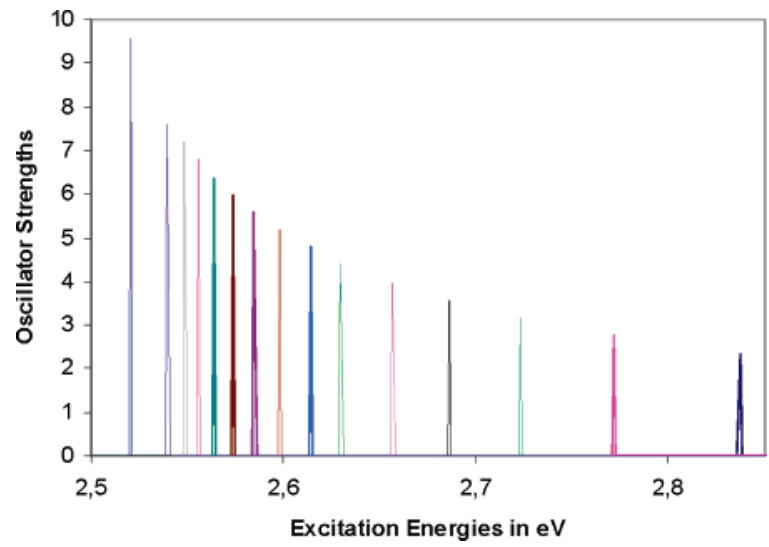

Figure 4. First allowed absorption peaks for neutral thiophene oligomers with 7-20 and 25 thiophene rings at the TDHF/ CEP-31G* level.

vertical excitation energies calculated for planar thiophene oligomers in the gas phase should lie in the vicinity of experimental RT solution and definitely above matrix data. Experimental ( $\lambda_{\max }$ values) and theoretical excitation energies are compared in Figure 3.

The CASPT2 ${ }^{37}$ value for bithiophene seems to be a bit low, lying between experimental RT solution and matrix values. Terthiophene was treated with a reduced active space since $18 \pi$ electrons are still too many to be treated fully. ${ }^{37}$ This might explain the severe underestimation of the excitation energy since the value is below the matrix data despite the absence of a solvent in the calculations. Note that the CASPT2 value lies also below the TDDFT result for 3T. The TDDFT value agrees with CASPT2 for bithiophene, but for longer oligomers, TDDFT excitation energies fall off faster than the experimental values and approach the matrix data already for $4 \mathrm{~T}$. The results would be useless in the long chain limit. In contrast, TDHF values appear to be very accurate. This confirms that TDHF is the best method, especially for long oligomers, to estimate the maximum absorption in long thiophene oligomers.

Figure 4 shows the progression of TDHF excitation energies and oscillator strengths for $7 \mathrm{~T}-25 \mathrm{~T}$. Spectra of all 
Table 1. Excitation Energies in eV, Oscillator Strength, and Main Electron Configurations in the Excited States (Weight \% for CASPT2, CI Coefficients for TDDFT) for the Bithiophene Cation at CASPT2 ${ }^{37}$ and TDDFT Levels of Theory

\begin{tabular}{|c|c|c|c|c|}
\hline & $1{ }^{2} \mathrm{~A}_{\mathrm{u}}$ & $2^{2} \mathrm{~B}_{\mathrm{g}}$ & $2{ }^{2} \mathrm{~A}_{\mathrm{u}}$ & $3{ }^{2} A_{u}$ \\
\hline \multirow[t]{4}{*}{ CASPT2 ${ }^{37}$} & $1.54(0.010)$ & $1.78(-)$ & $1.95(0.062)$ & $2.78(0.607)$ \\
\hline & $\mathrm{H}-1 \rightarrow \mathrm{H} 63 \%$ & $\mathrm{H}-2 \rightarrow \mathrm{H} 75 \%$ & $\mathrm{H}-3 \mathrm{H} \rightarrow 54 \%$ & $\mathrm{H} \rightarrow \mathrm{L} 44 \%$ \\
\hline & $\mathrm{H} \rightarrow \mathrm{L} 9 \%$ & & $H \rightarrow L 18 \%$ & $\mathrm{H}-3 \rightarrow \mathrm{H} 18 \%$ \\
\hline & & & $\mathrm{H}-1 \rightarrow \mathrm{H} 7 \%$ & $\mathrm{H}-1 \rightarrow \mathrm{H} 5 \%$ \\
\hline \multirow[t]{3}{*}{ TDBP86 } & $1.55(0.002)$ & $1.74(-)$ & $2.15(0.029)$ & $3.23(0.463)$ \\
\hline & $\mathrm{H}-1 \rightarrow \mathrm{H} 1.00$ & $\mathrm{H}-2 \rightarrow \mathrm{L} 1.00$ & $\mathrm{H}-3 \rightarrow \mathrm{H} 0.78$ & $\mathrm{H} \rightarrow \mathrm{L} 0.71$ \\
\hline & & & $\mathrm{H} \rightarrow \mathrm{L} 0.65$ & $\mathrm{H}-3 \rightarrow \mathrm{H} 0.46$ \\
\hline \multirow[t]{4}{*}{ TDB3P86-30\% } & $1.94(0.002)$ & $2.14(-)$ & $2.25(0.061)$ & $3.23(0.477)$ \\
\hline & $\mathrm{H}-1 \rightarrow \mathrm{H} 0.97$ & $\mathrm{H}-3 \rightarrow \mathrm{L} 1.00^{a}$ & $\mathrm{H}-2 \rightarrow \mathrm{H} 0.84$ & $\mathrm{H} \rightarrow \mathrm{L} 0.77$ \\
\hline & $\mathrm{H}-2 \rightarrow \mathrm{H} 0.25$ & & $\mathrm{H} \rightarrow \mathrm{L} 0.60$ & $\mathrm{H}-2 \rightarrow \mathrm{H}-0.47$ \\
\hline & $\mathrm{H} \rightarrow \mathrm{L} 0.23$ & & $\mathrm{H}-1 \rightarrow \mathrm{H} 0.29$ & $\mathrm{H}-2 \rightarrow \mathrm{H} 0.25$ \\
\hline in $\mathrm{CH}_{2} \mathrm{Cl}_{2}$ & $1.95(0.003)$ & $2.16(-)$ & $2.21(0.11)$ & $3.06(0.54)$ \\
\hline expt. ${ }^{b}$ & & & 2.10 (weak) & 2.92 (strong) \\
\hline expt. ${ }^{c}$ & & & 2.14 (weak) & 2.95 (strong) \\
\hline
\end{tabular}

${ }^{a}$ That $\mathrm{H}-2$ and $\mathrm{H}-3$ are reversed with TDBP86 as compared to TDB3P86-30\% is due to the near degeneracy of these energy levels. The near degeneracy is caused by the crossing of the states arising from HOMO and HOMO-1 levels of the thiophene repeat unit (compare ref 48 ). ${ }^{b}$ Electron absorption in freon glass at $77 \mathrm{~K}$, ref 36. ${ }^{c}$ Electron absorption in acetonitrile in the presence of TCNE, ref 25.

species are dominated by one strong absorption peak that shifts to lower energy and increases in oscillator strengths as chain lengths increase. The peak is dominated by the highest occupied molecular orbital-lowest unoccupied molecular orbital (HOMO-LUMO) transition with a coefficient of about 0.6 for short oligomers. At long chain lengths, the coefficient of the leading excitation decreases (to 0.39 for 25T), as lower- and higher-lying orbitals start contributing. For long oligomers, there are also some additional weaker features with oscillator strengths of $\sim 1 / 10$ that of the strong peak and at $0.2-0.4 \mathrm{eV}$ higher energies. The weak features are not included in Figure 4 for clarity. The difference between the excitation energies of $19 \mathrm{~T}$ and $20 \mathrm{~T}$ is $0.009 \mathrm{eV}$. Adding five more rings decreases the excitation energy by $0.02 \mathrm{eV}$ to a value of $2.52 \mathrm{eV}$ for 25T. Experimentally, there is no more change in the excitation energies beyond 20T, while absorption intensities and conductivities keep increasing in going from $20 \mathrm{~T}$ to $27 \mathrm{~T} .^{11}$

Cations. Assessment of the TDDFT Results. Since the performance of unrestricted (U)TDDFT excited-state calculations for radical cations of conjugated systems is not wellestablished, the accuracy of the results is investigated by comparing the $2 \mathrm{~T}^{+}$and $3 \mathrm{~T}^{+}$data with CASPT2 results. ${ }^{37}$ Rubio et al. reported the four lowest excited states for $2 \mathrm{~T}^{+}$ and the five lowest excited states for $3 \mathrm{~T}^{+}$. Like the present TDDFT results, CASPT2 excitation energies were obtained on ground-state structures of the radical cations and correspond to vertical excitation energies in the gas phase. Structure optimizations were done at the UB3PW91/ccpVDZ level of theory. The main difference between the structures obtained at B3P86-30\%/CEP-31G* and the ones reported by Rubio et al. is that all bonds are about $0.01 \AA$ longer with B3P86-30\%/CEP-31G*. Comparison with B3P86$30 \% / 6-31 \mathrm{G}^{*}$ structures shows that the bond length increase is caused by the pseudopotentials. B3P86-30\%/CEP-31G* structures of polyenes are, however, in close agreement with experimental results. ${ }^{41}$

Following the same nomenclature as Rubio et al., ${ }^{37}$ energy levels are labeled HOMO, HOMO-1, LUMO, LUMO+1, and so on. In a CASSCF wave function, there are no orbital energies, but states and orbitals can be matched by comparing their symmetries. The wave function of the ion was shown to be dominated by a single electron configuration obtained by removing one electron from the HOMO of the neutral species. This electron configuration has a weight of $84 \%$ for $2 \mathrm{~T}^{+}$and $79 \%$ for $3 \mathrm{~T}^{+}$in the CASSCF wave function. The term "HOMO" is also used for radical cations in which the HOMO is then only half-occupied. The HOMO (semioccupied molecular orbital, SOMO) corresponds to polaron level 1 (pol1) in solid-state nomenclature. All excited states are dominated by single excitations, doubles having total contributions between 4 and $7 \% .{ }^{37}$ This justifies the use of a single-electron method like TDDFT.

In open-shell DFT calculations, separate spin-orbitals are obtained for electrons with $\alpha$ and $\beta$ spin. Upon ionization, one electron with $\beta$ spin is removed. For radical cations, $\alpha$ and $\beta$ orbitals have different energies. The half-occupied HOMO (SOMO) of the cation in the CASPT2 wave function corresponds to the pair of the HOMO of the $\alpha$ electrons and the LUMO of the $\beta$ electrons in the unrestricted TDDFT formalism. HOMO- 1 is the pair of $\alpha$ and $\beta$ orbitals lying below the HOMO/LUMO pair and so on. In this way, the CASPT2 states and UTDDFT orbitals can be matched although the energies of $\alpha$ - and $\beta$-spin orbitals are not identical. Excited states arise as combinations of singleelectron transitions within the $\alpha$ - and $\beta$-orbital spaces. Since there are no transitions between $\alpha$ - and $\beta$-orbital spaces, the energetic shift of $\alpha$ versus $\beta$ orbitals is irrelevant for analyzing the nature of the electronic states. A thorough comparison of the composition of the CASPT2 wave functions ${ }^{37}$ for the excited states with the configurations contributing to the excited states in UTDDFT calculations revealed a close correspondence. UTDDFT and CASPT2 excited states of $2 \mathrm{~T}^{+}$are compared in Table 1 . Note that the electron configurations are given as weight percents for CASPT2, while the TDDFT values are CI coefficients.

The first feature in the spectrum at the CASPT2 level arises from a linear combination of the HOMO- $1 \rightarrow$ HOMO 


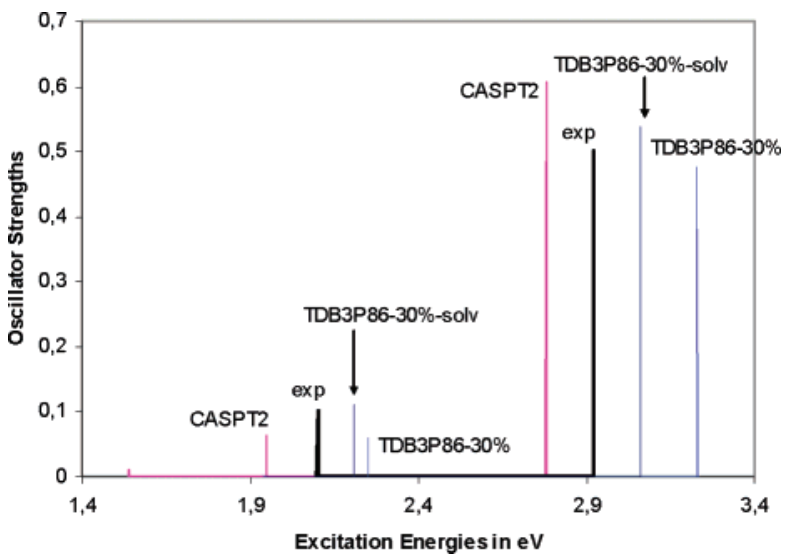

Figure 5. Excitation spectra for $2 \mathrm{~T}^{+}$compared to experimental results at the CASPT2 and TDB3P86-30\% levels of theory. The experimental oscillator strengths were arbitrarily set to 0.1 and 0.5 .

$(\mathrm{H}-1 \rightarrow \mathrm{H})$ and $\mathrm{HOMO} \rightarrow$ LUMO $(\mathrm{H} \rightarrow \mathrm{L})$ transitions with the same sign. This leads to partial cancellation of the electric dipole transition moment vectors. ${ }^{71}$ Since the two transition energies differ for OT radical cations, in contrast to those in polyene radical cations, ${ }^{71}$ the first excited state is dominated by the $\mathrm{H}-1 \rightarrow \mathrm{H}$ transition. As a result, the cancellation of the electric dipole transition moment vectors is incomplete and there is oscillator strength in this first peak. The strong (fourth) peak arises from the linear combination of the same two transitions with opposite signs and is dominated by the $\mathrm{H} \rightarrow \mathrm{L}$ transition. The opposite sign combination leads to addition of the electric dipole transition moment vectors and a higher oscillator strength. The third peak arises from the $\mathrm{H}-3 \rightarrow \mathrm{H}$ transition with some contributions from $\mathrm{H}-1 \rightarrow \mathrm{H}$ and $\mathrm{H} \rightarrow \mathrm{L}$ transitions. Rubio at al. ${ }^{37}$ assigned peaks 3 and 4 to the experimentally observed absorptions.

The same four lowest excited states as predicted by CASPT2 are also found with UTDDFT calculations. The TDDFT excitation energies obtained with the hybrid functional are between 0.3 and $0.4 \mathrm{eV}$ higher than that at CASPT2; the nature of the excited states and the oscillator strengths match qualitatively. There is a very close agreement for the two higher-energy peaks between TDBP86 and TDB3P86-30\% results. The general trend is that pure TDDFT finds some of the peaks at lower energy than the TDDFT hybrid. It is therefore clear that TDDFT produces qualitatively correct results with hybrid and pure DFT functionals.

To probe the solvent effect, TDDFT-hybrid calculations were carried out in the presence of dichloromethane, a common solvent for OT radical cations. ${ }^{16,26}$ Changing the solvent is not expected to lead to different results. ${ }^{74}$ The solvent hardly influences the low-energy transitions but lowers the strong absorption peak in energy. This brings the TDDFT-hybrid results in close agreement with experimental results. Figure 5 shows that CASPT2 gas-phase values are slightly lower than experimental excitation energies. Inclusion of a solvent would therefore deteriorate the agreement with experimental results. Thus, TDDFT-hybrid results seem to match experimental results at least as well and maybe better than CASPT2 values.
A similar comparison as for $2 \mathrm{~T}^{+}$is summarized for $3 \mathrm{~T}^{+}$ in Table 2. In addition, the effect of a counterion in the absence and in the presence of a solvent has been examined for $3 \mathrm{~T}^{+}$. In contrast to $2 \mathrm{~T}^{+}$, the $\mathrm{H}-3 \rightarrow \mathrm{L}$ transition leads to a peak with very low oscillator strength for $3 \mathrm{~T}^{+}$. At the same time, the first peak, the linear combination of the $\mathrm{H}-1 \rightarrow \mathrm{H}$ and $\mathrm{H} \rightarrow \mathrm{L}$ transition with same sign, gains oscillator strength. Thus, the first visible peaks in the spectra of $2 \mathrm{~T}^{+}$ and $3 \mathrm{~T}^{+}$are of different electronic origin. The trends regarding the excitation energies are the same as for $2 \mathrm{~T}^{+}$. CASPT2 values are lower than gas-phase data; TDB3P86$30 \%$ are higher. TDBP86 values lie in between but closer to TDB3P86-30\%. The difference between the strong peaks with and without $\mathrm{HF}$ exchange is about $0.1 \mathrm{eV}$. Inclusion of a solvent tends to lower excitation energies. The influence of the counterion, especially in the presence of the solvent, is very small.

Interesting is the splitting of the strong absorption peak predicted by CASPT2. ${ }^{37}$ This splitting is reproduced with TDBP86-30\% but not with TDBP86. It also depends on the medium as the splitting disappears with a counterion and reappears when the solvent is added. We will see below that peak splitting appears only for $3 \mathrm{~T}^{+}$and vanishes for longer OT radical cations. TDBP86 produces splitting for $4 \mathrm{~T}^{+}$, which also vanishes for all other chain lengths. Thus, it seems that the splitting of the strong peak into two closely spaced features is caused by subtle configuration mixing and depends on the energy levels, which in turn depend on chain length. This theoretical "fine structure" might not be observable in the experiment. Figure 6 summarizes the results and shows that TDDFT is very reliable and probably more accurate than CASPT2.

Chain-Length Dependence of Excitation Spectra. Having established the reliability of the TDDFT results, the chain-length dependence of the excitation energies can be investigated. Excited states in the energy range below the TDHF excitation energies of the neutral species were obtained at the TDB3P86-30\%/CEP-31G* and TDBP86/ CEP-31G* levels of theory. The results are summarized in Table 3 and compared to experimental values where available. Weak features with oscillator strengths below 0.3 are omitted unless they correspond to peaks that grow in intensity with increasing chain lengths.

For oligomers with two to six six-rings, TDDFT predicts two sub-band transitions arising from the combination of $\mathrm{H}-1 \rightarrow \mathrm{H}$ and $\mathrm{H} \rightarrow \mathrm{L}$ transitions with the same and with opposite signs. The low-energy feature has low oscillator strength but increases in intensity upon chain-length extension as the $\mathrm{H}-1 \rightarrow \mathrm{H}$ transition becomes more dominant in the excited-state wave function. The increase in intensity with increasing chain length is in agreement with experimental results. ${ }^{16}$ TDB3P86-30\% and TDBP86 results are in close agreement with each other, overestimating experimental values by up to $0.28 \mathrm{eV}$ (neglecting solvent effects). Solvent effects on excitation energies evaluated at the TDBP86 level for $8 \mathrm{~T}^{+}$are smaller than for $2 \mathrm{~T}^{+}$and $3 \mathrm{~T}^{+}, 0.11 \mathrm{eV}$, and bring the excitation energies to within $0.1-0.2 \mathrm{eV}$ of the experimental values. Although solvents effects have a small 
Table 2. Excitation Energies in eV, Oscillator Strength, and Main Electron Configurations in the Excited States (Weight \% for CASPT2, CI Coefficients for TDDFT) for the Terthiophene Cation at CASPT2 ${ }^{37}$ and TDDFT Levels of Theory

\begin{tabular}{|c|c|c|c|c|c|}
\hline & $1^{2} \mathrm{~B}_{1}$ & $2^{2} \mathrm{~B}_{1}$ & $3^{2} \mathrm{~B}_{1}$ & $2{ }^{2} \mathrm{~A}_{2}$ & $3^{2} \mathrm{~A}_{2}$ \\
\hline \multirow[t]{4}{*}{ CASPT2 $2^{37}$} & $1.31(0.051)$ & $1.94(0.643)$ & $2.12(0.351)$ & $2.21(0.002)$ & $2.50(<0.001)$ \\
\hline & $\mathrm{H}-1 \rightarrow \mathrm{H} 47 \%$ & $H \rightarrow L 28 \%$ & $\mathrm{H}-2 \rightarrow \mathrm{H} 51 \%$ & $\mathrm{H}-3 \rightarrow \mathrm{L} 63 \%$ & $\mathrm{H}-4 \rightarrow \mathrm{L} 38 \%$ \\
\hline & $H \rightarrow L 24 \%$ & $\mathrm{H}-1 \rightarrow \mathrm{H} 23 \%$ & $\mathrm{H} \rightarrow \mathrm{L} 16 \%$ & & $\mathrm{H} \rightarrow \mathrm{L}+119 \%$ \\
\hline & & $\mathrm{H}-2 \rightarrow \mathrm{H} 14 \%$ & & & $\mathrm{H}-1 \rightarrow \mathrm{L} 16 \%$ \\
\hline \multirow[t]{3}{*}{ TDBP86 } & $1.52(0.038)$ & $2.56(0.84)$ & $1.73(0.023)$ & $1.76(<0.001)$ & $2.14(-)$ \\
\hline & $\mathrm{H}-1 \rightarrow \mathrm{H} 0.75$ & $\mathrm{H} \rightarrow \mathrm{L} 0.71$ & $\mathrm{H}-2 \rightarrow \mathrm{H} 0.93^{\mathrm{a}}$ & $\mathrm{H}-3 \rightarrow \mathrm{L} 1.00$ & $\mathrm{H}-4 \rightarrow \mathrm{L} 0.99$ \\
\hline & $\mathrm{H} \rightarrow \mathrm{L} 0.59$ & $\mathrm{H}-1 \rightarrow \mathrm{H}-0.40$ & & & \\
\hline \multirow[t]{4}{*}{ TDB3P86-30\% } & $1.65(0.076)$ & $2.51(0.480)$ & $2.62(0.313)$ & $2.22(0.120)$ & $2.27(0.001)$ \\
\hline & $\mathrm{H}-1 \rightarrow \mathrm{H} 0.85$ & $\mathrm{H} \rightarrow \mathrm{L} 0.59$ & $\mathrm{H}-4 \rightarrow \mathrm{H} 0.76^{a}$ & $\mathrm{H}-2 \rightarrow \mathrm{H} 0.93$ & $\mathrm{H}-3 \rightarrow \mathrm{H} 0.97$ \\
\hline & $\mathrm{H} \rightarrow \mathrm{L} 0.63$ & $\mathrm{H}-1 \rightarrow \mathrm{H} 0.28$ & $\mathrm{H} \rightarrow \mathrm{L} 0.51$ & & \\
\hline & & $\mathrm{H}-4 \rightarrow \mathrm{H} 0.61$ & $\mathrm{H}-1 \rightarrow \mathrm{H} 0.28$ & & \\
\hline TDB3P86-30\% in $\mathrm{CH}_{2} \mathrm{Cl}_{2}$ & $1.65(0.146)$ & $2.21(0.442)$ & $2.42(0.387)$ & $2.33(0.002)$ & $2.56(0.076)$ \\
\hline with $\mathrm{Cl}_{3}^{-}$ & $1.75(0.057)$ & $2.49(0.033)$ & $2.61(0.658)$ & $2.19(0.094)$ & $2.37(0.003)$ \\
\hline with $\mathrm{Cl}_{3}{ }^{-}$in $\mathrm{CH}_{2} \mathrm{Cl}_{2}$ & $1.67(0.129)$ & $2.20(0.386)$ & $2.43(0.430)$ & $2.36(0.003)$ & $2.55(0.087)$ \\
\hline Expt. ${ }^{b}$ & 1.46 (weak) & 2.25 (strong) & & & \\
\hline
\end{tabular}

a That $\mathrm{H}-2, \mathrm{H}-3$, and $\mathrm{H}-4$ are reversed with TDBP86 as compared to TDB3P86-30\% is due to the near degeneracy of these energy levels. The near degeneracy is caused by the crossing of the states arising from HOMO and HOMO-1 levels of the thiophene repeat unit (compare ref 48). ${ }^{b}$ Electron absorption in acetonitrile ref 22.

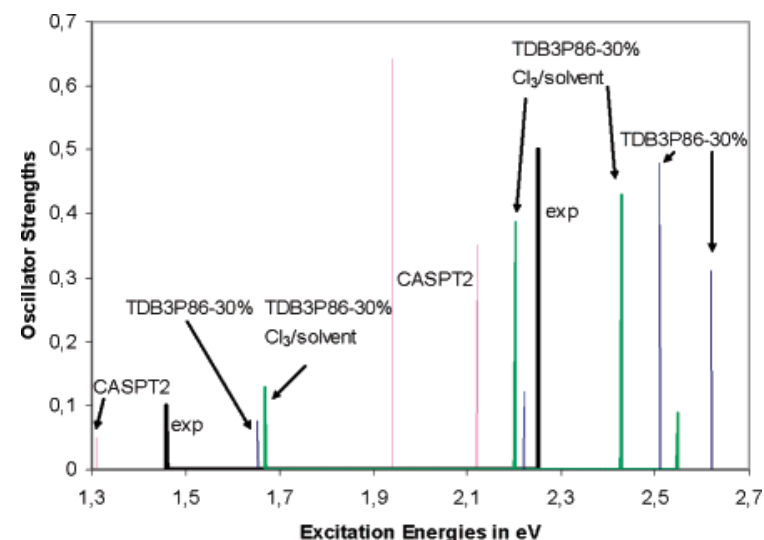

Figure 6. Excitation spectra for $3 \mathrm{~T}^{+}$compared to experimental results at the CASPT2 and TDDFT levels of theory. The experimental oscillator strengths were arbitrarily set to 0.1 and 0.5 .

influence on the excitation energies, there are significant shifts in oscillator strengths.

For $8 \mathrm{~T}^{+}$, the first peak in the spectrum consists almost entirely of the $\mathrm{H}-1 \rightarrow \mathrm{H}$ electron configuration. The second peak originates from the $\mathrm{H} \rightarrow \mathrm{L}$ transition, but additional electronic configurations contribute. Due to this configuration mixing, the $\mathrm{H} \rightarrow \mathrm{L}$ transition splits into two separate peaks at 1.45 and at $2.56 \mathrm{eV}$. The new peak at $2.56 \mathrm{eV}$ has a low oscillator strength for $8 \mathrm{~T}^{+}$but increases rapidly in intensity with increasing chain lengths while a fourth sub-band absorption that appears at $2.74 \mathrm{eV}$ vanishes in the long chain limit. Stick spectra for $2 \mathrm{~T}^{+}$through $8 \mathrm{~T}^{+}$at TDB3P86-30\% are compared in Figure 7. Some differences start to arise between TDB3P86-30\% and TDBP86 predictions as the second absorption in the $8 \mathrm{~T}^{+}$spectrum is split into two closely spaced $(\sim 0.14-0.15 \mathrm{eV})$ peaks and the third transition lies lower in energy and has a higher oscillator strength.

For $12 \mathrm{~T}^{+}$, the two additional sub-band transitions lie at 2.14 and $2.47 \mathrm{eV}$, slightly below the TDHF interband transition of neutral $12 \mathrm{~T}$ at $2.63 \mathrm{eV}$. Due to configuration mixing, the lower component of the $\mathrm{H} \rightarrow \mathrm{L}$ transition (second peak) that dominates spectra of short oligomers decreases in intensity with increasing chain length. Starting with $12 \mathrm{~T}^{+}$, the $\mathrm{H}-1 \rightarrow \mathrm{H}$ transition and the higher component of the $\mathrm{H}$ $\rightarrow$ L transition have higher oscillator strengths than the original $\mathrm{H} \rightarrow \mathrm{L}$ transition. The contributions of various electronic transitions to the three main peaks at TDB3P86$30 \%$ are represented graphically in Figure 8 for $12 \mathrm{~T}^{+}$. Transitions with coefficients above 0.3 were considered. It might be confusing that the HOMO- 1 of the $\beta$ electrons lies above the HOMO of the $\alpha$ electrons in Figure 8. As described above, the energetic shift between $\alpha$ and $\beta$ energy levels can be ignored, since no transitions occur between $\alpha$ and $\beta$ energy levels. The semioccupied HOMO according to Rubio et al.' $s^{37}$ nomenclature corresponds therefore still to the $\alpha-\mathrm{HOMO} / \beta$-LUMO pair of spin-orbitals. In Figure $8, \mathrm{Ru}-$ bio's nomenclature is applied and the $\alpha-\mathrm{HOMO} / \beta$-LUMO pair is designated as HOMO for $\alpha$ and $\beta$ electrons. Therefore, the first transition at $0.46 \mathrm{eV}$, which is dominated by a single electron transition, is the $\mathrm{H}-1 \rightarrow \mathrm{H}$ transition. Peaks 2 and 3 have strong $\mathrm{H} \rightarrow \mathrm{L}$ contributions but mix with $\mathrm{H}-1 \rightarrow \mathrm{L}+1$ and $\mathrm{H}-2 \rightarrow \mathrm{L}$ transitions.

For $14 \mathrm{~T}^{+}$and longer OT cations, the higher-lying component of the $\mathrm{H} \rightarrow \mathrm{L}$ transition (peak 3 ) becomes the dominant peak in the spectrum. The second peak, which was the strongest for short oligomers, is the weakest in the long chain length limit. Figure 9 shows that a huge absorption is predicted for peak 3 at about $0.5 \mathrm{eV}$ below the interband transition of the neutral species. Using geometries with localized defects produces the sub-band transition with almost the same energy but with about half of the oscillator strength. Nonetheless, even with a localized defect, the third peak remains the strongest absorption in the spectrum for long oligomers. The fourth peak in the $2.4-2.7 \mathrm{eV}$ region that is rather intense for $10 \mathrm{~T}^{+}$through $14 \mathrm{~T}^{+}$is absent for $16 \mathrm{~T}^{+}$and $19 \mathrm{~T}^{+}$.

Since TDB3P86-30\% and TDP86 spectra differ in the high-energy sub-band region of longer OT cations, stick spectra for $8 \mathrm{~T}^{+}$through $20 \mathrm{~T}^{+}$are plotted at the TDBP86 
Table 3. Excitation Energies in eV and Oscillator Strengths (in Parenthesis) for Thiophene Oligomer Cations

\begin{tabular}{|c|c|c|c|c|c|}
\hline & method & E1 & E2 & E3 & E4 \\
\hline \multirow[t]{3}{*}{$4 T^{+}$} & TDB3P86-30\% & $1.34(0.18)$ & $2.11(1.22)$ & & \\
\hline & TDBP86 & $1.22(0.10)$ & $2.09(0.26)$ & $2.17(0.94)$ & \\
\hline & expt. ${ }^{16}$ & 1.16 & 1.92 & & \\
\hline \multirow[t]{3}{*}{$5 \mathrm{~T}^{+}$} & TDB3P86-30\% & $1.12(0.33)$ & $1.86(1.53)$ & & \\
\hline & TDBP86 & $1.01(0.18)$ & $1.86(1.38)$ & & \\
\hline & expt. ${ }^{16}$ & 0.98 & 1.72 & & \\
\hline \multirow[t]{4}{*}{$6 \mathrm{~T}^{+}$} & TDB3P86-30\% & $0.96(0.53)$ & $1.68(1.70)$ & & \\
\hline & TDBP86 & $0.88(0.29)$ & $1.66(1.64)$ & & \\
\hline & expt. ${ }^{16}$ & 0.84 & 1.59 & & \\
\hline & expt. $^{34}$ & 0.83 & 1.58 & & \\
\hline \multirow[t]{3}{*}{$8 \mathrm{~T}^{+}$} & TDB3P86-30\% & $0.74(1.00)$ & $1.45(1.75)$ & $2.56(0.16)$ & $2.74(0.11)$ \\
\hline & TDBP86 & $0.70(0.57)$ & $1.39(0.81)$ & $1.53(0.21)$ & $2.16(0.63)$ \\
\hline & in $\mathrm{CH}_{2} \mathrm{Cl}_{2}$ & $0.64(0.29)$ & $1.28(1.70)$ & $1.43(0.56)$ & $2.18(0.53)$ \\
\hline \multirow[t]{3}{*}{$9 \mathrm{~T}^{+}$} & TDB3P86-30\% & $0.65(1.23)$ & $1.37(1.67)$ & $2.42(0.38)$ & $2.68(0.09)$ \\
\hline & TDBP86 & $0.65(0.73)$ & $1.29(0.88) /$ & $1.36(0.83)$ & $2.00(0.78)$ \\
\hline & expt. $^{32}$ & 0.67 & 1.46 & & \\
\hline \multirow[t]{2}{*}{$9 \mathrm{~T}-\mathrm{Cl}_{3}$} & TDB3P86-30\% & $0.86(0.84)$ & $1.47(1.67)$ & $1.71(0.31)$ & not calculated \\
\hline & in $\mathrm{CH}_{2} \mathrm{Cl}_{2}$ & $0.76(1.36)$ & $1.37(1.64)$ & $1.67(0.12)$ & \\
\hline \multirow[t]{2}{*}{$10 \mathrm{~T}^{+}$} & TDB3P86-30\% & $0.58(1.44)$ & $1.30(1.55)$ & $2.30(0.65)$ & $2.63(0.32)$ \\
\hline & TDBP86 & $0.57(0.86)$ & $1.28(1.41)$ & $1.86(0.46)$ & $1.88(0.75)$ \\
\hline \multirow[t]{3}{*}{$12 \mathrm{~T}^{+}$} & TDB3P86-30\% & $0.46(1.76)$ & $1.21(1.21)$ & $2.14(1.38)$ & $2.47(0.55)$ \\
\hline & TDBP86 & $0.48(1.14)$ & $1.18(1.35)$ & $1.69(1.04)$ & $1.70(0.38)$ \\
\hline & expt. $^{32}$ & $0.59 / 0.62$ & $1.42 / 1.45$ & 2.68 & \\
\hline $13 \mathrm{~T}^{+}$ & TDB3P86-30\% & $0.41(1.86)$ & $1.17(1.07)$ & $2.08(1.90)$ & $2.41(0.54)$ \\
\hline $13 \mathrm{~T}^{+} / / 13 \mathrm{~T}-\mathrm{Cl}_{3}$ & TDB3P86-30\% & $0.45(1.87)$ & $1.19(1.44)$ & $2.03(0.90) / 2.28(0.28)$ & $2.45(0.72)$ \\
\hline \multirow[t]{4}{*}{$13 \mathrm{~T}-\mathrm{Cl}_{3}$} & TDB3P86-30\% & $0.77(1.31)$ & $1.24(0.88)$ & $1.58(1.52)$ & $2.60(0.58)$ \\
\hline & in $\mathrm{CH}_{2} \mathrm{Cl}_{2}$ & $0.64(1.83)$ & $1.21(1.03)$ & $1.50(0.92)$ & $2.34(0.33) /$ \\
\hline & & & & & $2.49(0.99) /$ \\
\hline & & & & & $2.58(0.40)$ \\
\hline \multirow[t]{2}{*}{$14 \mathrm{~T}^{+}$} & TDB3P86-30\% & $0.37(1.93)$ & $1.14(0.93)$ & $2.05(2.47)$ & $2.38(0.60)$ \\
\hline & TDBP86 & $0.40(1.32)$ & $1.15(1.15)$ & $1.57(1.39) / 1.59(0.65)$ & $2.06(0.76)$ \\
\hline \multirow[t]{3}{*}{$16 \mathrm{~T}^{+}$} & TDB3P86-30\% & $0.31(1.99)$ & $1.09(0.71)$ & $2.00(3.54)$ & \\
\hline & TDBP86 & $0.34(1.44)$ & $1.12(0.94)$ & $1.48(1.62)$ & \\
\hline & & & & $1.50(0.66)$ & \\
\hline $19 \mathrm{~T}^{+}$ & TDB3P86-30\% & $0.24(1.99)$ & $1.05(0.50)$ & $1.95(5.12)$ & \\
\hline $19 \mathrm{~T}^{+} / / 19 \mathrm{~T}-\mathrm{Cl}_{3}$ & TDB3P86-30\% & $0.27(2.21)$ & $0.99(0.64)$ & $1.86(2.75)$ & $2.17(1.36)$ \\
\hline \multirow[t]{2}{*}{$19 \mathrm{~T}-\mathrm{Cl}_{3}$} & TDB3P86-30\% & $0.71(1.50)$ & $1.07(0.35)$ & $1.34(1.30)$ & $1.46(0.56)$ \\
\hline & & & $1.63(0.95)$ & $2.29(1.67)$ & $2.37(1.20)$ \\
\hline \multirow[t]{2}{*}{$20 \mathrm{~T}^{+}$} & TDB3P86-30\% & $0.23(1.97)$ & $1.03(0.44)$ & $1.95(5.61)$ & \\
\hline & TDBP86 & $0.26(1.56)$ & $1.11(0.71)$ & $1.37(1.92) / 1.40(0.55)$ & \\
\hline
\end{tabular}

level in Figure 10. The prediction of additional sub-band transitions is confirmed with TDBP86. However, with pure TDDFT, several of the peaks are split into multiple components. The third peak for $20 \mathrm{~T}^{+}$lies lower in energy than with the hybrid functional. In addition, the electronic configurations of the transitions are different, having less contribution from the $\mathrm{H} \rightarrow \mathrm{L}$ excitation and more contributions involving higher and lower energy levels. These findings seem to be consistent with the more compressed band structure produced with pure DFT. Considering the general performances of pure DFT and DFT-hybrid, showing that DFT-hybrid produces better band gaps, ${ }^{44,48-50}$ and more accurate relative energies of occupied orbitals,${ }^{51}$ it seems that the peak positions obtained with the hybrid functional are more reliable.
Effect of Counterions. Since experimental work showed that the doping process is similar no matter which dopant is used, ${ }^{109}$ only $\mathrm{Cl}_{3}{ }^{-}$counterions were employed here. The structure of $13 \mathrm{~T}-\mathrm{Cl}_{3}$ is shown in Figure 11. The charge transfer from the thiophene chain to the counterion is $0.96 e$ in $9 \mathrm{~T}-\mathrm{Cl}_{3}$, in $13 \mathrm{~T}-\mathrm{Cl}_{3}$, and in $19 \mathrm{~T}-\mathrm{Cl}_{3}$ according to natural bond order analysis. ${ }^{110}$ The complete charge transfer indicates that OT cations and counterions form ion pairs that interact electrostatically.

As shown in Figure 2 for $19 \mathrm{~T}-\mathrm{Cl}_{3}$, the positive charge is highest in the three central rings. The charges decrease gradually toward the second to last ring from the chain end and increase somewhat in the terminal ring. $\mathrm{Cl}_{3}$ complexes have therefore localized defects in terms of structure and electron distribution with defect sizes of about 11 rings. $9 \mathrm{~T}^{+}$ 


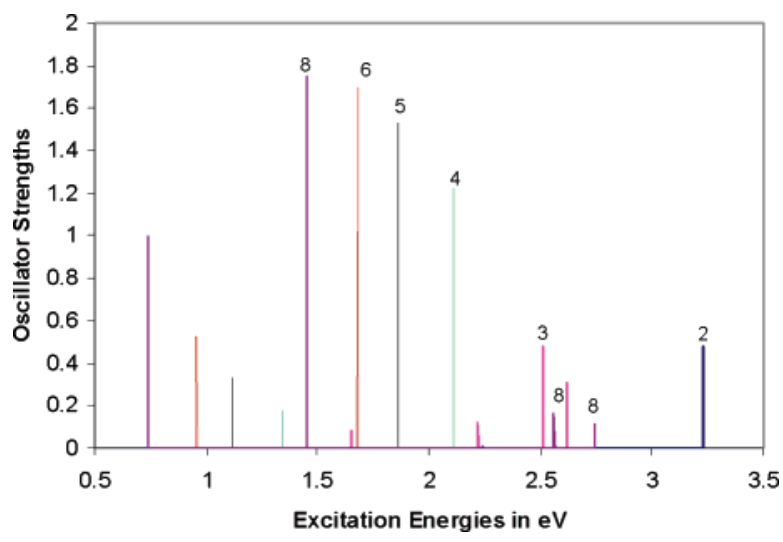

Figure 7. Stick spectra for $2 \mathrm{~T}^{+}$through $8 \mathrm{~T}^{+}$at the TDB3P86$30 \%$ level.

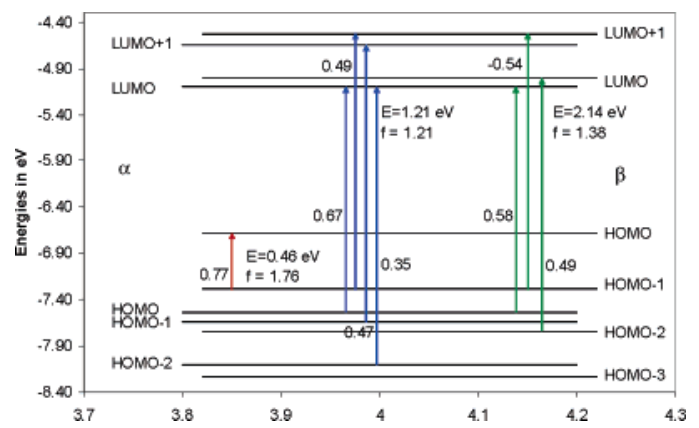

Figure 8. Electronic configurations contributing to the three strong sub-band transitions in $12 \mathrm{~T}^{+}$at the TDB3P86-30\% level. The numbers close to the arrows give the coefficients of the transitions. $\beta$-electron energy levels are shifted right with respect to the $\alpha$ levels. The orbitals are labeled according to Rubio et al.'s ${ }^{37}$ nomenclature, which is described in the text.

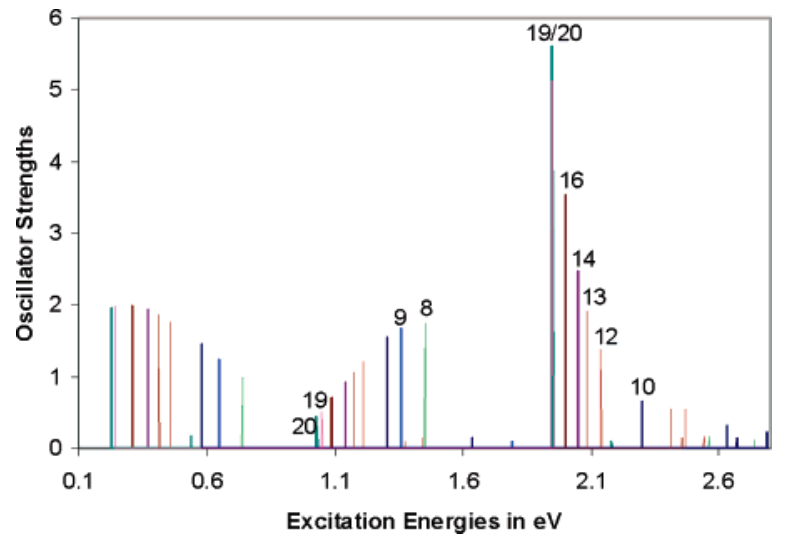

Figure 9. Stick spectra for $8 \mathrm{~T}^{+}$through $20 \mathrm{~T}^{+}$at the TDB3P86$30 \%$ level.

is therefore too short for having a converged defect, and $13 \mathrm{~T}^{+}$ has only about the size of the localized defect. For $19 \mathrm{~T}^{+}$, true localization effects can be expected.

TDB3P86-30\% excitation energies for $9 \mathrm{~T}-\mathrm{Cl}_{3}, 13 \mathrm{~T}-\mathrm{Cl}_{3}$, and $19 \mathrm{~T}-\mathrm{Cl}_{3}$ are included in Table 3. Compared to $9 \mathrm{~T}^{+}$, the excitation energies of $9 \mathrm{~T}-\mathrm{Cl}_{3}$ shift as follows. The first excited state lies $0.21 \mathrm{eV}$ higher in energy, and its oscillator strength is reduced from 1.23 to 0.84 . The second excitation energy is increased by $0.10 \mathrm{eV}$ without a change in oscillator strength. The third excited state lies $0.72 \mathrm{eV}$ lower in energy with a similar oscillator strength as in the absence of the

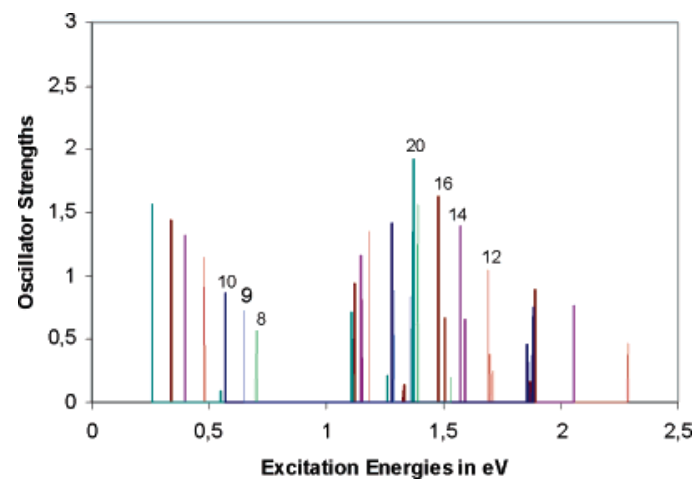

Figure 10. Stick spectra for $8 \mathrm{~T}^{+}$through $20 \mathrm{~T}^{+}$at the TDBP86 level.

counterion. Thus, the main effect of the counterion is lowering of the third peak. There are only small changes in excitation energies and the oscillator strength when a solvent is considered.

Compared to $13 \mathrm{~T}^{+}$, the first excitation energy of $13 \mathrm{~T}-\mathrm{Cl}_{3}$ increases by $0.36 \mathrm{eV}$. The energy of the second transition increases by $0.06 \mathrm{eV}$. Both peaks decrease in oscillator strength. The third transition lies $0.5 \mathrm{eV}$ lower in the presence of a counterion. The electronic configurations contributing to these states are depicted in Figure 12. One of the electron configurations contributing to the third peak directly involves the counterion. This explains the strong effect of the counterion on this transition. Otherwise, there is little change in the nature of the absorption peaks compared to $12 \mathrm{~T}^{+}$ (Figure 8). Removing the counterion without letting the structure relax results in absorption energies that differ by less than $0.05 \mathrm{eV}$ from those of $13 \mathrm{~T}^{+}$. The changes between $13 \mathrm{~T}^{+}$and $13 \mathrm{~T}-\mathrm{Cl}_{3}$ are therefore not due to structural localization but caused by changes in the electron distribution. Adding a solvent splits the peak that arises in the 2.4$2.6 \mathrm{eV}$ region of the cation and complex into three components. Otherwise, the influence of the solvent on the spectrum is small.

The inclusion of a counterion increases the energies of the first two peaks of $19 \mathrm{~T}-\mathrm{Cl}_{3}$ by 0.47 and $0.02 \mathrm{eV}$ compared to $19 \mathrm{~T}^{++}$. Instead of the strong peak at $1.95 \mathrm{eV}$, there are five absorptions between 1.3 and $2.3 \mathrm{eV}$ in the presence of the counterion. The two peaks at 1.07 and $1.34 \mathrm{eV}$ have $\mathrm{H}$ $\rightarrow \mathrm{L}$ contributions but are multiconfigurational. The two features at 1.46 and $1.63 \mathrm{eV}$ have major contributions (coefficients of 0.55 and 0.69 ) from transitions that originate from a $\mathrm{Cl}_{3}{ }^{-}$orbital (HOMO-6). The peaks at 2.29 and 2.37 $\mathrm{eV}$ originate from $\mathrm{H}-1 \rightarrow \mathrm{L}+1$ and $\mathrm{H} \rightarrow \mathrm{L}+2$ transitions. Removing the counterion reverses the changes to a large extent as three peaks are predicted for structurally localized $19 \mathrm{~T}^{+} / / 19 \mathrm{~T}-\mathrm{Cl}_{3}$ that differ by only up to $0.09 \mathrm{eV}$ from those of $19 \mathrm{~T}^{+}$. The biggest difference between $19 \mathrm{~T}^{+}$with a delocalized defect and $19 \mathrm{~T}^{+}$with a structurally localized defect is that the third peak loses oscillator strength, 5.12 versus 2.75. Thus, the effect of structural localization is moderate; the effect of the counterion is substantial, since it is directly involved in some of the electronic transitions. This latter effect might differ with other counterions. In general, the spectral region of the third peak is very sensitive to 


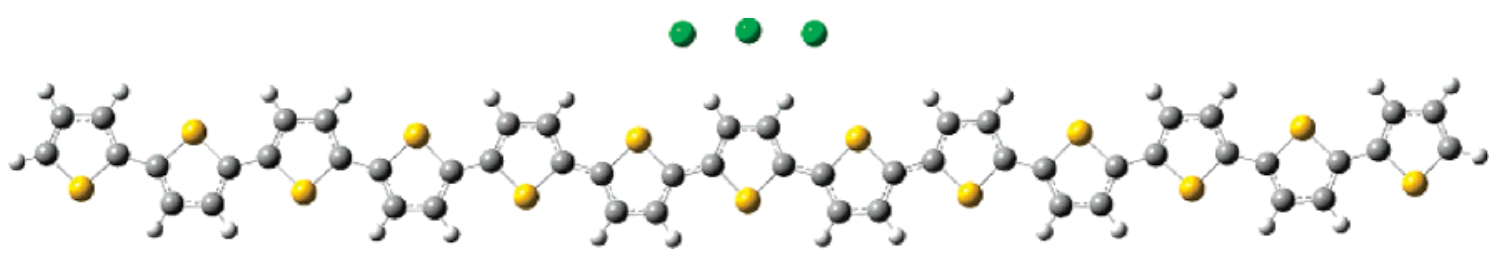

Figure 11. Optimized structure of $13 \mathrm{~T}-\mathrm{Cl}_{3}$.

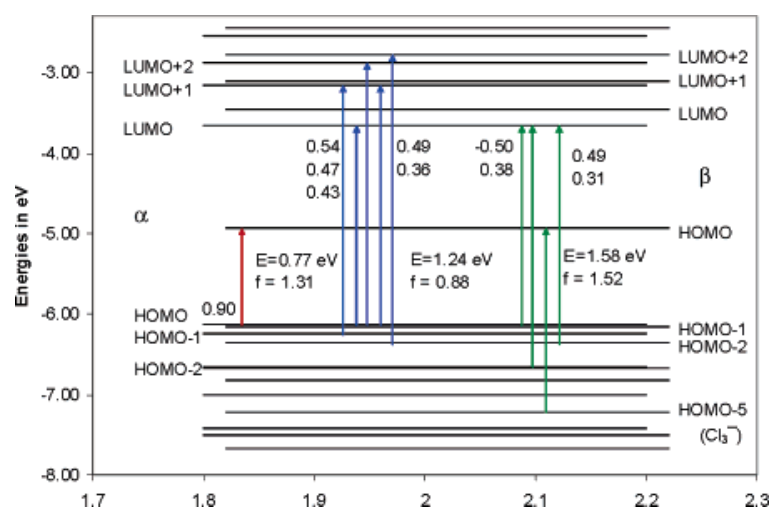

Figure 12. Electronic configurations contributing to the three strong sub-band transitions in $13 \mathrm{~T}-\mathrm{Cl}_{3}$ at the TDB3P $86-30 \%$ level. The numbers close to the arrows give the coefficients of the transitions. $\beta$-electron energy levels are shifted right with respect to the $\alpha$ levels.

changes in the method and in the geometry and to the presence of a counterion and a solvent. This region of the spectrum requires further study.

Comparison between Theory and Experiment. For short OT cations, a comparison between theory and experiment is straightforward as the two bands predicted by theory match experimental values well. The influence of counterions is small, and solvent effects lower the theoretical excitation energies, improving the agreement between theory and experiment. As the chains get longer, theory predicts a third band that increases rapidly in oscillator strength. This subband transition appears at a chain length of eight rings; for $12 \mathrm{~T}^{+}$, three peaks of comparable oscillator strength are predicted. Doping of $12 \mathrm{~T}$ with perchlorate in dichloromethane $e^{32}$ and of $13 \mathrm{~T}$ with $\mathrm{FeCl}_{3}$ in dichloromethane ${ }^{34}$ was reported. The bands of the $13 \mathrm{~T}$ cation at 0.85 and $1.67 \mathrm{eV}$ are close to those at 0.88 and $1.75 \mathrm{eV}$ for the second doping stage of $12 \mathrm{~T}$. Since these values are close to those for $6 \mathrm{~T}^{+}$, the features were attributed to two polarons located on the same chain. Haare et al., ${ }^{32}$ however, reported an earlier doping stage with bands at $0.59-0.62$ and $1.42-1.45 \mathrm{eV}$. These bands belong most likely to the monocations and are used for comparison here. The theoretical predictions of 0.46 and $1.21 \mathrm{eV}$ for $12 \mathrm{~T}^{+}$and 0.45 and $1.19 \mathrm{eV}$ for $13 \mathrm{~T}^{+}$are $\mathrm{a}$ little lower than the experimental values.

In addition, to the two well-known features comparably intense peaks are predicted at 2.14 and $2.08 \mathrm{eV}$ for $12 \mathrm{~T}^{+}$ and $13 \mathrm{~T}^{+}$. The appearance of these additional peaks close to the interband transition of the neutral oligomer is important as doping experiments on $12 \mathrm{~T}^{32}$ showed that, in contrast to shorter OTs, the interband transition did not disappear but was reduced to half its size. This was rationalized in terms of a disproportionation of $12 \mathrm{~T}^{+}$into neutral $12 \mathrm{~T}$ and the dication. The present results indicate that the unexpected peak may be the third peak of the radical cation. Similar findings were reported in a theoretical and experimental study by Grozema et al. ${ }^{97}$ As chain lengths increase further, the third peak is predicted to dominate the spectrum of the radical cations. Such a peak has never been discussed explicitly in experimental studies, but the spectra presented by Nakanishi at al. ${ }^{34}$ for $41 \mathrm{~T}^{+}$show a strong absorption slightly below that of the neutral oligomer at low doping levels. These findings will need further analysis.

\section{Discussion}

Comparison with experimental and CASPT2 results shows that TDDFT-hybrid excitation energies and oscillator strengths are reliable and almost quantitatively correct. For short oligothiophene cations, TDDFT employing hybrid and pure DFT functionals leads to very similar excitation spectra. The same agreement was obtained for closed-shell polyene cations. ${ }^{41}$ In contrast, hybrid functionals do not work for polyene radical cations because of large spin contaminations. For long chain systems, pure TDDFT and TDDFT-hybrid predictions completely agree for the low-energy peaks, and both predict an additional strong sub-band transition. Pure TDDFT tends, however, to place certain peaks lower in energy than TDDFT-hybrid. Since pure DFT produces too small band widths and too small energy gaps, TDDFT-hybrid is probably more reliable.

The appearance of two low-energy peaks in PT instead of one in polyacetylene (PA) ${ }^{41}$ can be traced back to the different $\mathrm{H}-1 \rightarrow \mathrm{H}$ and $\mathrm{H} \rightarrow \mathrm{L}$ energy gaps. For both systems, there exist, in principle, two excited states which arise from combinations of $\mathrm{H}-1 \rightarrow \mathrm{H}$ and $\mathrm{H} \rightarrow \mathrm{L}$ with the same and with opposite signs. ${ }^{36,37,71}$ The combination with the same signs leads to cancellation of the electric dipole transition moment vectors and has a low oscillator strength. For polyene radical cations, the cancellation is almost complete since the energy of the two transitions is practically the same. Thus, the first transition is not observed. For OTs, the cancellation is incomplete because the energy-level difference is not the same. As a result, the low-energy excited state is dominated by $\mathrm{H}-1 \rightarrow \mathrm{H}$ and has oscillator strength. The highenergy excited state is dominated by $\mathrm{H} \rightarrow \mathrm{L}$. For mediumsized OTs with around 10 rings, both excited states have similar oscillator strengths. Thus, the different numbers of peaks in PA and PT do not correspond to different localized species (solitons vs bipolarons) as predicted by the polaron model.

The polaron model ${ }^{5,111}$ was developed on the basis of calculations applying the Su-Schrieffer-Heeger (SSH) Hamiltonian. ${ }^{112}$ According to this model, localized intragap 


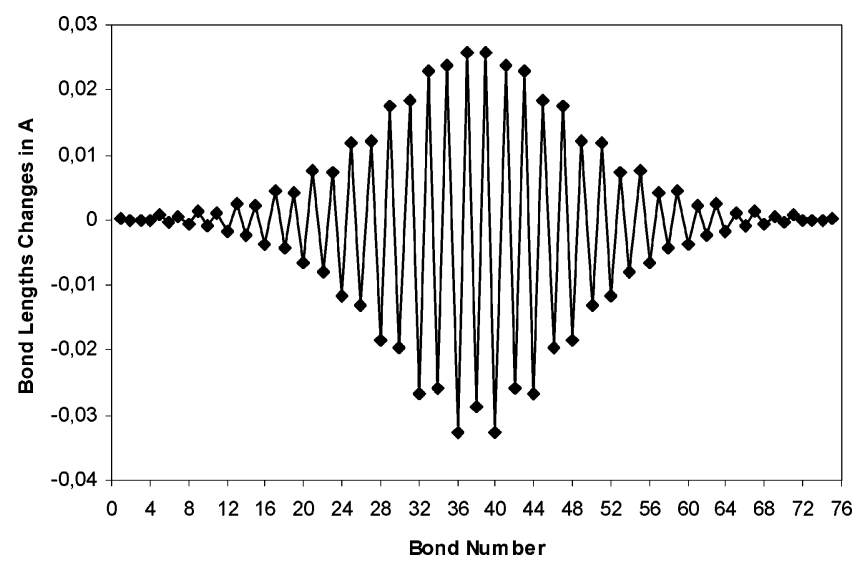

Figure 13. Bond length changes in $12 \mathrm{~T}^{+}$compared to neutral $12 \mathrm{~T}$ at the BHandHLYP/CEP-31G* level of theory.

states arise from kinks (solitons) or bound kink-antikink pairs (polarons), both of which extend over 15-20 lattice spacings. Big emphasis is therefore placed on the "selflocalization" of defects. ${ }^{4}$ Applied to polypyrrole, localization over four rings was obtained for polarons at the SSH level. ${ }^{113}$ Localized defects were reproduced for polyene cations in the absence of counterions at the restricted modified neglect of diatomic overlap (MNDO) level "modified to deal with open shell systems (by placing " $1 / 2$ electron" of each spin in the open shell ...)". ${ }^{114}$ A polaron size of four rings was also determined for PT with the $\mathrm{SSH}^{115}$ method and with MNDO calculations on $6 \mathrm{~T}^{+} .6$ Thiophene cations with up to nine rings were optimized at the semiempirical AM1 level, employing restricted open-shell Hartree-Fock (ROHF) wave functions ${ }^{38}$ because ROHF "better reproduces the localization of the charge carriers along the chain as compared to the unrestricted unrestricted Hartree-Fock (UHF) approach."116 An upper limit for the defect size of five rings was estimated in this way. It was shown more recently, however, that, while it is true that UHF overestimates the width of solitons of neutral polyenes, ROHF underestimates it. ${ }^{117}$ Localized defects were also obtained with two-configuration SCF. ${ }^{54}$ Defect localization was confirmed for thiophene cations with UMP2 and DFT-hybrid (BHandHLYP), ${ }^{98}$ in contrast to pure DFT results. ${ }^{118}$

The problem with ab initio and HF-based semiempirical calculations on polarons is that wave functions of open-shell species are spin-contaminated, and the problem grows as the chains get longer. Therefore, one must choose between spincontaminated and restricted wave functions, the former leading to delocalized defects, the latter to localized ones. The above summary shows that the calculations concerning defect sizes have been adjusted in one way or another to reproduce the localized defects predicted with the $\mathrm{SSH}$ method. Localization of polarons has therefore never been proven at higher levels of theory. Here, it is found that openshell DFT-hybrid calculations produce delocalized defects in the absence of spin-contamination.

Since the localized defects at the BHandHLYP/3-21G* level ${ }^{98}$ are at odds with the findings of this work, geometries of $12 \mathrm{~T}, 12 \mathrm{~T}^{+}$, and $19 \mathrm{~T}^{+}$were optimized at the BHandHLYP/ CEP-31G* level. The bond length changes upon ionization are shown in Figure 13 for $19 \mathrm{~T}^{+}$. While it is true that the

\section{Scheme 1}

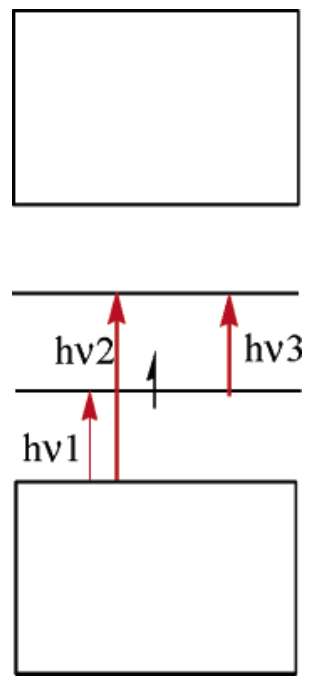

defect is more localized with BHandHLYP than with B3P86$30 \%$, the defect still spreads over all but the last two rings in $19 \mathrm{~T}^{+}$. This corresponds to a defect size of 15 rings. The energy lowering during the optimization starting with the B3P86-30\% geometry is $1.96 \mathrm{kcal} / \mathrm{mol}$. Thus, the potential energy surface is confirmed to be very flat, and highly accurate calculations would be required to determine the geometry with certainty. The half and half (HandH) functional includes $50 \% \mathrm{HF}$ exchange. Therefore, it does not come as a surprise that the expectation values of the spinoperator are 1.15 for $12 \mathrm{~T}^{+}$and 1.30 for $19 \mathrm{~T}^{+}$. Increasing the amount of HF exchange to $50 \%$ simply reintroduces the spin-contamination problem of ab initio methods. The BHandHLYP geometry is therefore not reliable and is no proof for polaron localization.

Apart from the problem with spin-contamination, HF calculations lack dynamic correlation. Since the driving force for defect localization is supposed to be the energy difference between quinoid and aromatic structures, Moro et al. ${ }^{96}$ tested the performance of HF, MP2, and DFT with respect to this energy difference for $2 \mathrm{~T}$. It turned out that HF overestimates the preference for the aromatic form by $30 \mathrm{kcal} / \mathrm{mol}$ compared to MP2 and BLYP, which predict preferences of 15.5 and $13 \mathrm{kcal} / \mathrm{mol}$, respectively. In agreement with these results, DFT-hybrid defect sizes were shown to agree with MP2 ones for solitons in odd-numbered polyene cations. ${ }^{41}$ Thus, DFT-B3P86-30\% geometries do not suffer from overdelocalization of defects compared to MP2. This discussion suggests that DFT-hybrid geometries are reliable for conjugated systems and that the delocalized defects predicted for radical cations are not a DFT artifact. Additional evidence against defect localization comes from a comparison of INDO/SCI excitation energies obtained at ROHF/AM1 and DFT structures, which shows that the originally very poor agreement with experimental results ${ }^{38,116}$ improves when delocalized DFT structures are employed. ${ }^{97}$

The delocalized nature of the defect agrees with earlier pure DFT results, ${ }^{118-120}$ which showed, moreover, that any defect localization decreases further in a three-dimensional lattice compared to isolated chains. Thus, the most accurate calculations performed on polarons to this date suggest that 


\section{$390909090909096 \%$}

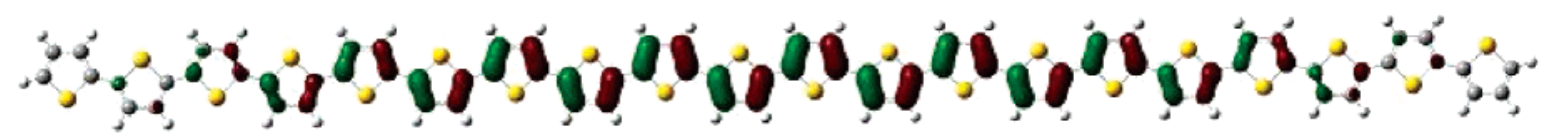

Figure 14. HOMO orbitals of $12 \mathrm{~T}$ and $20 \mathrm{~T}$ at the B3P86-30\% level of theory.

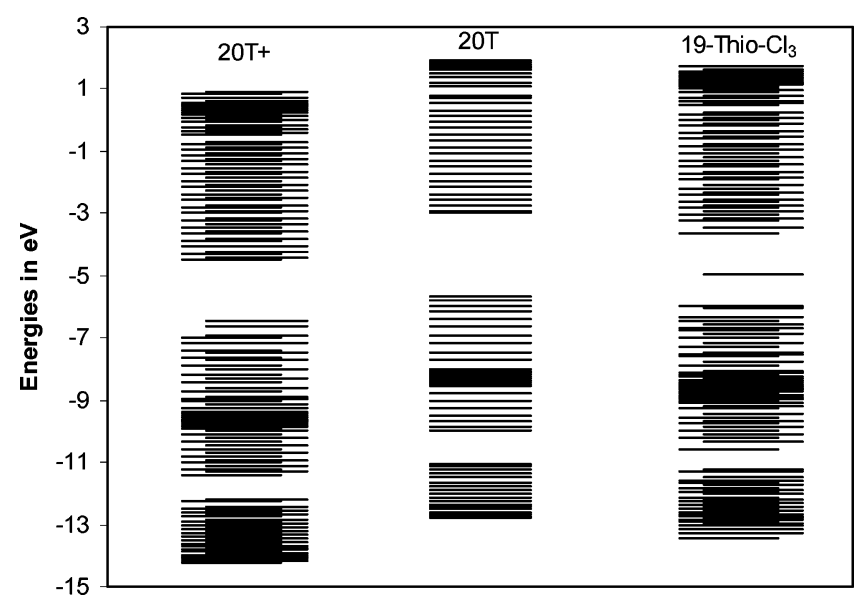

Figure 15. $\pi$-orbital energies of $20 \mathrm{~T}, 20 \mathrm{~T}^{+}$, and $19 \mathrm{~T}-\mathrm{Cl}_{3}$ at the UTDB3P86-30\% level of theory. $\beta$-orbital energies are shifted right with respect to $\alpha$ levels.

these defects are delocalized. This is exactly what qualitative molecular orbital theory predicts. As the electron is removed from a molecular orbital that is delocalized almost over the entire molecule, except for the terminal rings (compare Figure 14), the finding of a delocalized defect that involves all but the terminal rings makes perfect sense. Why should ionization involve only five thiophene rings? Experimental evidence for localization is necessarily obtained in the presence of counterions. In the presence of counterions, DFT predicts defect localization over 11 rings.

According to the polaron model, oligothiophene cations should have two new energy levels placed symmetrically in the band gap. These gap states are believed to be a consequence of the formation of a geometrically localized defect upon ionization. The two intragap states give rise to three sub-band transitions (Scheme 1): $h \nu 1$ forms a valence band to HOMO, $\mathrm{h} v 2$ forms a valence band to the second intragap state, and $\mathrm{h} v 3$ forms a band between the two intragap states.

In Figure 15 , the $\pi$-orbital energies are plotted for $20 \mathrm{~T}$, $20 \mathrm{~T}^{+}$, and $19 \mathrm{~T}-\mathrm{Cl}_{3}$. Due to their chain length, these cations may serve as models for polymers. Although the meaning of DFT orbital energies is a matter of debate, ${ }^{121-123}$ a comparison of energy levels obtained with B3LYP and peaks in ultraviolet photoelectron spectra revealed agreement regarding relative energies of the occupied orbitals although the ionization potentials applying Koopmans's theorem are too low. ${ }^{51}$ Combined with the accurate band gaps predicted at the B3P86-30\% level, ${ }^{44}$ orbital energy plots should give rather accurate relative positions of the occupied and unoccupied energy levels. Figure 15 does not confirm the energy-level diagram as shown in Scheme 1. There are no two symmetrically placed levels in the gap for the cation with and without counterions. For $20 \mathrm{~T}^{+}$, the highest $\beta$ level at $-6.42 \mathrm{eV}$ is empty and belongs to the lower polaron level together with the highest occupied $\alpha$ orbital at $-6.96 \mathrm{eV}$. The other $\beta$ level at $-6.62 \mathrm{eV}$ is occupied. Thus, pol1 is very close to the valence band. pol 2 is merged with the conduction band. This might be attributed to the lack of defect localization. In the presence of the counterion, and therefore with defect localization, only the empty $\beta$ level moves up. There is still no pol2 in the gap.

Despite the absence of the two intragap states, TDDFT correctly predicts sub-band transitions. These transition are obtained at virtually the same energies with delocalized and with localized defects. Thus, defect localization cannot be the reason for the sub-band transitions. Analysis of the nature of these excited states at the CASPT2 and TDDFT level shows that the $\mathrm{h} v 1$ and $\mathrm{h} v 3$ transitions, which correspond to $\mathrm{H}-1 \rightarrow \mathrm{H}$ and $\mathrm{H} \rightarrow \mathrm{L}$ transitions, couple with the same and opposite signs to give rise to a weak low-energy and a strong higher-energy absorption peak. Scheme 1 is therefore inconsistent with the electronic nature of these first two excited states. Moreover, the $\mathrm{H} \rightarrow \mathrm{L}$ transition couples with additional transitions especially in the long chain limit. The last transition $\mathrm{h} v 2$, corresponding to $\mathrm{H}-1 \rightarrow \mathrm{L}$, is not predicted at the CASPT2 and TDDFT levels of theory.

\section{Conclusions}

Although TDDFT has problems with excited states of neutral conjugated $\pi$ systems, TDDFT gives very good results for conjugated cations and radical cations. This conclusion is based on a comparison of TDDFT excitation energies with CASPT2 data on $2 \mathrm{~T}^{+}$and $3 \mathrm{~T}^{+}$and with experimental results for long OT radical cations. While hybrid functionals produce large spin contamination for polyene radical cations, spin contamination is virtually absent in open-shell calculations on oligothiophene radical cations. Therefore, the more reliable hybrid functionals can be used for OT radical cations.

For short- to medium-sized oligomers, two sub-band transitions are obtained. In the long chain limit, TDDFT predicts one additional intense sub-band transition. Experimental data on $12 \mathrm{~T}^{+}$and $41 \mathrm{~T}^{+}$seem to confirm the existence of such a transition close to the interband transition of the neutral system.

The two lower-energy sub-band features of OT radical cations have the same electronic origin as the single subband peak in polyene radicals and radical cations. In both systems of $\mathrm{H}-1 \rightarrow \mathrm{H}$ and $\mathrm{H} \rightarrow \mathrm{L}$, transitions mix with the same and opposite signs. With equal transition energies as 
in polyene radical cations, the low-energy peak has no oscillator strength and the high-energy peak is very strong. With different excitation energies as in OT radical cations, the low-energy peak becomes visible. Thus, polarons may give rise to one, two, or three sub-band peaks depending on the system and on the conjugation length.

DFT methods predict delocalized defects in the absence of spin contamination. Usually, the reliability of DFT methods regarding this issue is questioned since DFT tends to overestimate conjugation. One should not forget, however, that the polaron model is based on Hückel-type calculations that lack a self-consistent treatment of Coulomb repulsions. Localized defects are only reproduced with semiempirical and ab initio methods that lack dynamic electron correlation and are based on restricted open-shell wave functions. Since the potential energy surfaces are very flat, most of the discrepancies between the results with different methods are caused by the small energy changes that are associated with distortions of the structures.

Spectra obtained for radical cations with optimized structures and with nonoptimized structures containing a localized structural defect are very similar. This proves beyond any doubt that defect localization is not the reason for the occurrence of sub-band transitions.

Spectra calculated in the presence of counterions differ especially for longer systems from those of bare cations due to a change in the electron density and due to direct involvement of the counterion in some of the electronic transitions.

With all due caution that should be applied when approximate methods are used and only isolated molecules are investigated, it must be stated that the present results are in contradiction with almost every aspect of the polaron model. Instead of the strong self-localization, there are very flat potential energy surfaces. The same sub-band transitions are obtained in the absence as in the presence of defect localization. The nature of the electronic transitions is more complicated than predicted with the polaron model as all of the peaks involve a combination of several electronic configurations. In the long chain limit, due to the closely spaced orbital energies, extensive configuration mixing contributes to the excited states. Care is therefore recommended when using the polaron model.

Acknowledgment. This work is supported by TÜBITAK (TBAG-2461) and by Bilkent University. I would like to thank Dennis Salzner for writing a program that extracts orbital energies from Gaussian 03 output files for plotting.

\section{References}

(1) Skotheim, T. A. Handbook of Conducting Polymers; Marcel Dekker: New York, 1986.

(2) Chung, T.-C.; Kaufman, J. H.; Heeger, A. J.; Wudl, F. Phys. Rev. B: Condens. Matter Mater. Phys. 1984, 30, 702.

(3) Brédas, J. L.; Thémans, B.; Fripiat, J. G.; André, J.-M.; Chance, R. R. Phys. Rev. B: Condens. Matter Mater. Phys. 1984, 29, 6761.

(4) Brédas, J. L.; Street, G. B. Acc. Chem. Res. 1985, 18, 309.
(5) Heeger, A. J.; Kivelson, S.; Schrieffer, J. R.; Su, W.-P. Rev. Mod. Phys. 1988, 60, 781.

(6) Stafström, S.; Brédas, J. L. Phys. Rev. B: Condens. Matter Mater. Phys. 1988, 38, 4180.

(7) Patil, A. O.; Heeger, A. J.; Wudl, F. Chem. Rev. 1988, 88, 183.

(8) Electronic Materials: The Oligomer Approach; Müllen, K., Wegener, G., Eds.; Wiley-VCH: Weinheim, Germany, 1997.

(9) Taliani, C.; Blinov, L. M. Adv. Mater. 1996, 8, 353.

(10) Fichou, D.; Teulade-Fichou, M.-P.; Horowitz, G.; Demanze, F. Adv. Mater. 1997, 9, 75.

(11) Nakanishi, H.; Sumi, N.; Aso, Y.; Otsubo, T. J. Org. Chem. 1998, 63, 8632 .

(12) Otsubo, T.; Nakanishi, H.; Aso, Y. Synth. Met. 1999, 101, 604.

(13) Sumi, N.; Nakanishi, H.; Ueno, S.; Takimiya, K.; Aso, Y.; Otsubo, T. Bull. Chem. Soc. Jpn. 2001, 74, 979.

(14) Otsubo, T.; Aso, Y.; Takimiya, K. Bull. Chem. Soc. Jpn. 2001, 74, 1789.

(15) Fichou, D.; Horowitz, G.; Garnier, F. Synth. Met. 1990, 39, 125.

(16) Fichou, D.; Horowitz, G.; Xu, B.; Garnier, F. Synth. Met. 1990, 39, 243.

(17) Guay, J.; Diaz, A.; Wu, R.; Tour, J. M. J. Am. Chem. Soc. 1993, 115, 1869.

(18) Guay, J.; Kasai, P.; Diaz, A.; Wu, R.; Tour, J. M.; Dao, L. H. Chem. Mater. 1992, 4, 1097.

(19) Hill, M. G.; Mann, K. R.; Miller, L. L.; Penneau, J.-F. J. Am. Chem. Soc. 1992, 114, 2728.

(20) Hill, M. G.; Penneau, J.-F.; Zinger, B.; Mann, K. R.; Miller, L. L. Chem. Mater. 1992, 4, 1106.

(21) Bäuerle, P.; Segelbacher, U.; Maier, A.; Mehring, M. J. Am. Chem. Soc. 1993, 115, 10217.

(22) Wintgens, V.; Valat, P.; Garnier, F. J. Phys. Chem. 1994, 98, 228.

(23) Yu, Y.; Gunic, E.; Zinger, B.; Miller, L. L. J. Am. Chem. Soc. 1996, 118, 1013.

(24) Zinger, B.; Mann, K. R.; Hill, M. G.; Miller, L. L. Chem. Mater. 1992, 4, 1113.

(25) Evans, C. H.; Scaiano, J. C. J. Am. Chem. Soc. 1990, 112, 2694.

(26) Fichou, D.; Xu, B.; Horowitz, G.; Garnier, F. Synth. Met. 1991, 41, 463 .

(27) Guay, J.; Diaz, A.; Wu, R.; Tour, J. M.; Dao, L. H. Chem. Mater. 1992, 4, 254.

(28) Hotta, S.; Waragai, K. J. Phys. Chem. 1993, 97, 7427.

(29) Horowitz, G.; Yassar, A.; von Bardeleben, H. J. Synth. Met. 1994, 62, 245.

(30) Furukawa, Y. J. Phys. Chem. 1996, 100, 15644.

(31) Graf, D. D.; Duan, R. G.; Campbell, J. P.; Miller, L. L.; Mann, K. R. J. Am. Chem. Soc. 1997, 119, 5888.

(32) Haare, J. A. E. H.; Havinga, E. E.; van Dongen, J. L. J.; Janssen, R. A. J.; Cornil, J.; Brédas, J. L. Chem.-Eur. J. 1998, 4, 1509.

(33) Tol, A. J. W. Synth. Met. 1995, 74, 95. 
(34) Nakanishi, H.; Sumi, N.; Ueno, S.; Takimiya, K.; Aso, Y.; Otsubo, T.; Komaguchi, K.; Shiotani, M.; Ohta, N. Synth. Met. 2001, 119, 413.

(35) Nessakh, B.; Horowitz, G.; Garnier, F.; Deloffre, F.; Srivastava, P.; Yassar, A. J. Electroanal. Chem. 1995, 399, 97.

(36) Keszthelyi, T.; Grage, M. M.-L.; Offersgard, J. F.; Wilbrandt, R.; Svendsen, C.; Sonnich Mortensen, O.; Pedersen, J. K.; Jensen, H. J. A. J. Phys. Chem. A 2000, 104, 2808.

(37) Rubio, M.; Ortí, E.; Pou-Amerigo, R.; Merchán, M. J. Phys. Chem. A 2001, 105, 9788.

(38) Cornil, J.; Beljonne, D.; Brédas, J. L. J. Chem. Phys. 1995, 103,842 .

(39) Ye, A.; Shuai, Z.; Kwon, O.; Brédas, J. L.; Beljonne, D. J. Chem. Phys. 2004, 121, 5567.

(40) Zade, S. S.; Bendikov, M. Chem--Eur. J. 2006, in press.

(41) Salzner, U. J. Chem. Theory Comput. 2007, 3, 219.

(42) Becke, A. D. J. Chem. Phys. 1993, 98, 5648.

(43) Perdew, J. P. Phys. Rev. B: Condens. Matter Mater. Phys. 1986, 33, 8822 .

(44) Salzner, U.; Lagowski, J. B.; Pickup, P. G.; Poirier, R. A. J. Phys. Chem. 1998, 102, 2572.

(45) Stevens, W.; Basch, H.; Krauss, J. J. Chem. Phys. 1984, 81, 6026.

(46) Stevens, W. J.; Krauss, M.; Basch, H.; Jasien, P. G. Can. J. Chem. 1992, 70, 612.

(47) Salzner, U.; Lagowski, J. B.; Pickup, P. G.; Poirier, R. A. J. Comput. Chem. 1997, 18, 1943.

(48) Salzner, U.; Lagowski, J. B.; Poirier, R. A.; Pickup, P. G. Synth. Met. 1998, 96, 177.

(49) Kertesz, M.; Choi, C. H.; Yang, S. Chem. Rev. 2005, 105, 3448 .

(50) Choi, C. H.; Kertesz, M.; Karpfen, A. J. Chem. Phys. 1997, 107, 6712.

(51) Sanchez-Carrera, R. S.; Coropceanu, V.; da Silva, D. A.; Friedlein, R.; Osikowicz, W.; Murdey, R.; Suess, C.; Salaneck, W. R.; Bredas, J. L. J. Phys. Chem. B 2006, 110, 18904.

(52) Bauernschmitt, R.; Ahlrichs, R. Chem. Phys. Lett. 1996, 256, 454.

(53) Dierksen, M.; Grimme, S. J. Phys. Chem. A 2004, 108, 10225.

(54) Irle, S.; Lischka, H. J. Chem. Phys. 1997, 107, 3021.

(55) Salzner, U. Curr. Org. Chem. 2004, 8, 569.

(56) Bauernschmitt, R.; Ahlrichs, R.; Henrich, F. H.; Kappes, M. M. J. Am. Chem. Soc. 1998, 120, 5052.

(57) Hirata, S.; Head-Gordon, M.; Bartlett, R. J. J. Chem. Phys. 1999, 111, 10774.

(58) Hirata, S.; Head-Gordon, M. Chem. Phys. Lett. 1999, 302, 375.

(59) Hsu, C.-P.; Hirata, S.; Head-Gordon, M. J. Phys. Chem. A 2001, 105, 451.

(60) Della Sala, F.; Heinze, H. H.; Görling, A. Chem. Phys. Lett. 2001, 339, 343.

(61) Ma, J.; Li, S.; Liang, Y. Macromolecules 2002, 35, 1109.
(62) Hieringer, W.; van Gisbergen, S. J. A.; Baerends, E. J. J. Phys. Chem. A 2002, 106, 10380.

(63) Dreuw, A.; Head-Gordon, M. Chem. Rev. 2005, 105, 4009.

(64) Cai, Z.-L.; Sendt, K.; Reimers, J. R. J. Chem. Phys. 2002, $117,5543$.

(65) Sala, F. D.; Görling, A. Int. J. Quantum Chem. 2003, 91, 131.

(66) Parac, M.; Grimme, S. Chem. Phys. 2003, 292, 11.

(67) Dreuw, A.; Weisman, J. L.; Head-Gordon, M. J. Chem. Phys. 2003, 119, 2943.

(68) Dreuw, A.; Head-Gordon, M. J. Am. Chem. Soc. 2004, 126, 4007.

(69) Dierksen, M.; Grimme, S. J. Chem. Phys. 2004, 120, 3544.

(70) Salzner, U. Conjugated Organic Polymers: From Bulk to Molecular Wire. In Handbook of Theoretical and Computational Nanotechnology; Rieth, M., Schommers, W., Eds.; ASP: Valencia, CA, 2006; Vol. 8, pp 203.

(71) Kawashima, Y.; Nakayama, K.; Nakano, H.; Hirao, H. Chem. Phys. Lett. 1997, 267, 82.

(72) Luo, Y.; Ågren, H.; Stafström, S. J. Chem. Phys. 1994, 98, 7782.

(73) Luo, Y.; Ruud, K.; Norman, P.; Jonsson, D.; Ågren, H. J. Phys. Chem. B 1998, 102, 1710.

(74) Li, Y. Modeling Solvent Effects on Excitation Energies for Polyenes. Master's Thesis, Bilkent University, Ankara, Turkey, 2006.

(75) Grozema, F. C.; van Duijnen, P. T.; Siebbeles, L. D.; Goossens, A.; Leeuw, S. W. J. Phys. Chem. B 2004, 108, 16139.

(76) Miertus, S.; Scrocco, E.; Tomasi, J. Chem. Phys. 1981, 55, 117.

(77) Miertus, S.; Tomasi, J. Chem. Phys. 1982, 65, 239.

(78) Cossi, M.; Cammi, R.; Tomasi, J. Chem. Phys. Lett. 1996, $255,327$.

(79) Cancès, M. T.; Mennucci, B.; Tomasi, J. J. Chem. Phys. 1997, 107, 3032.

(80) Barone, V.; Cossi, M.; Tomasi, J. J. Chem. Phys. 1997, 107, 3210 .

(81) Cossi, M.; Barone, V.; Mennucci, B.; Tomasi, J. Chem. Phys. Lett. 1998, 286, 253.

(82) Barone, V.; Cossi, M.; Tomasi, J. J. Comput. Chem. 1998, 19, 404.

(83) Barone, V.; Cossi, M. J. Phys. Chem. A 1998, 102, 1995.

(84) Mennucci, B.; Tomasi, J. J. Chem. Phys. 1997, 106, 5151.

(85) Mennucci, B.; Cancès, M. T.; Tomasi, J. J. Phys. Chem. B 1997, 101, 10506.

(86) Tomasi, J.; Mennucci, B.; Cancès, M. T. THEOCHEM 1999, 464, 211.

(87) Cammi, R.; Mennucci, B.; Tomasi, J. J. Phys. Chem. A 1999, $103,9100$.

(88) Cossi, M.; Barone, V.; Robb, M. A. J. Chem. Phys. 1999, 111, 5295.

(89) Cammi, R.; Mennucci, B.; Tomasi, J. J. Phys. Chem. A 2000, 104, 5631.

(90) Cossi, M.; Barone, V. J. Chem. Phys. 2000, 112, 2427. 
(91) Cossi, M.; Barone, V. J. Chem. Phys. 2001, 115, 4708.

(92) Cossi, M.; Rega, N.; Scalmani, G.; Barone, V. J. Chem. Phys. 2001, 114, 5691.

(93) Cossi, M.; Scalmani, G.; Rega, N.; Barone, V. J. Chem. Phys. 2002, 117, 43.

(94) Cossi, M.; Rega, N.; Scalmani, G.; Barone, V. J. Comput. Chem. 2003, 24, 669.

(95) Frisch, M. J.; Trucks, G. W.; Schlegel, H. B.; Scuseria, G. E.; Robb, M. A.; Cheeseman, J. R.; Montgomery, J. A., Jr.; Vreven, T.; Kudin, K. N.; Burant, J. C.; Millam, J. M.; Iyengar, S. S.; Tomasi, J.; Barone, V.; Mennucci, B.; Cossi, M.; Scalmani, G.; Rega, N.; Petersson, G. A.; Nakatsuji, H.; Hada, M.; Ehara, M.; Toyota, K.; Fukuda, R.; Hasegawa, J.; Ishida, M.; Nakajima, T.; Honda, Y.; Kitao, O.; Nakai, H.; Klene, M.; Li, X.; Knox, J. E.; Hratchian, H. P.; Cross, J. B.; Bakken, V.; Adamo, C.; Jaramillo, J.; Gomperts, R.; Stratmann, R. E.; Yazyev, O.; Austin, A. J.; Cammi, R.; Pomelli, C.; Ochterski, J. W.; Ayala, P. Y.; Morokuma, K.; Voth, G. A.; Salvador, P.; Dannenberg, J. J.; Zakrzewski, V. G.; Dapprich, S.; Daniels, A. D.; Strain, M. C.; Farkas, O.; Malick, D. K.; Rabuck, A. D.; Raghavachari, K.; Foresman, J. B.; Ortiz, J. V.; Cui, Q.; Baboul, A. G.; Clifford, S.; Cioslowski, J.; Stefanov, B. B.; Liu, G.; Liashenko, A.; Piskorz, P.; Komaromi, I.; Martin, R. L.; Fox, D. J.; Keith, T.; Al-Laham, M. A.; Peng, C. Y.; Nanayakkara, A.; Challacombe, M.; Gill, P. M. W.; Johnson, B.; Chen, W.; Wong, M. W.; Gonzales, C.; Pople, J. A. Gaussian 03, Revision D.01; Gaussian, Inc.: Wallingford, CT, 2004.

(96) Moro, G.; Scalmani, G.; Cosentino, U.; Pitea, D. Synth. Met. 2000, 108, 165.

(97) Grozema, F. C.; Candeias, L. P.; Swart, M.; van Duijnen, P. T.; Wildeman, J.; Hadziioanou, G.; Siebbeles, L. D. A.; Warman, J. M. J. Chem. Phys. 2002, 117, 11366.

(98) Geskin, V. M.; Dkhissi, A.; Brédas, J. L. Int. J. Quantum Chem. 2003, 91, 350.

(99) Dkhissi, A.; Beljonne, D.; Lazzaroni, R.; Louwet, F.; Groenendaal, L.; Brédas, J. L. Int. J. Quantum Chem. 2003, 91, 517.

(100) Geskin, V. M.; Grozema, F. C.; Siebbeles, L. D. A.; Beljonne, D.; Brédas, J. L.; Cornil, J. J. Phys. Chem. B 2005, 109, 20237.

(101) Champagne, B.; Spassova, M. Phys. Chem. Chem. Phys. 2004, 6, 3167.

(102) Monev, V.; Spassova, M.; Champagne, B. Int. J. Quantum Chem. 2005, 104, 354.
(103) Martinez, F.; Voelkel, R.; Naegele, D.; Naarmann, H. Mol. Cryst. Liq. Cryst. 1989, 167, 227.

(104) Birnbaum, D.; Kohler, B. E. J. Chem. Phys. 1989, 90, 3506.

(105) Birnbaum, D.; Kohler, B. E. J. Chem. Phys. 1991, 95, 4783.

(106) Birnbaum, D.; Fichou, D.; Kohler, B. E. J. Chem. Phys. 1992, 96, 165.

(107) D'Amico, K. L.; Manos, C.; Christensen, R. L. J. Am. Chem. Soc. 1980, 102, 1777.

(108) Guillaume, M.; Champagne, B.; Zutterman, F. J. Phys. Chem. A 2006, 110, 13007.

(109) Fincher, C. R., Jr; Ozaki, M.; Heeger, A. J.; MacDiarmid, A. G. Phys. Rev. B: Condens. Matter Mater. Phys. 1979, 19, 4140.

(110) Reed, A. E.; Curtiss, L. A.; Weinhold, F. Chem. Rev. 1988, $88,899$.

(111) Fesser, K.; Bishop, A. R.; Campbell, D. K. Phys. Rev. B: Condens. Matter Mater. Phys. 1983, 27, 4804.

(112) Su, W. P.; Schrieffer, J. R.; Heeger, A. J. Phys. Rev. B: Condens. Matter Mater. Phys. 1980, 22, 2099.

(113) Brédas, J. L.; Scott, J. C.; Yakushi, K.; Street, G. B. Phys. Rev. B: Condens. Matter Mater. Phys. 1984, 30, 1023.

(114) Boudreaux, D. S.; Chance, R. R.; Brédas, J. L.; Silbey, R. Phys. Rev. B: Condens. Matter Mater. Phys. 1983, 28, 6927.

(115) Brédas, J. L.; Wudl, F.; Heeger, A. J. Solid State Commun. 1987, 63, 577.

(116) Cornil, J.; Beljonne, D.; Brédas, J. L. J. Chem. Phys. 1995, 103,834

(117) Bally, T.; Hrovat, D. A.; Thatcher Borden, W. Phys. Chem. Chem. Phys. 2000, 2, 3363.

(118) Brocks, G. Synth. Met. 1999, 102, 914.

(119) Vogl, P.; Campbell, C. K. Phys. Rev. Lett. 1989, 62, 2012.

(120) Vogl, P.; Campbell, C. K. Phys. Rev. B: Condens. Matter Mater. Phys. 1990, 41, 12797.

(121) Perdew, J. P.; Levy, M. Phys. Rev. Lett. 1983, 51, 1884.

(122) Baerends, E. J.; Gritsenko, O. V. J. Phys. Chem. A 1997, 101,5383 .

(123) Stowasser, R.; Hoffmann, R. J. Am. Chem. Soc. 1999, 121, 3414.

CT600344U 